JOURNAL OF THE

AMERICAN MATHEMATICAL SOCIETY

Volume 19, Number 2, Pages 461-486

S 0894-0347(05)00510-2

Article electronically published on November 15, 2005

\title{
OPERADS AND KNOT SPACES
}

\author{
DEV P. SINHA
}

\section{INTRODUCTION}

Let $E_{m}$ denote the space of embeddings of the interval $\mathbb{I}=[-1,1]$ in the cube $\mathbb{I}^{m}$ with endpoints and tangent vectors at those endpoints fixed on opposite faces of the cube, equipped with a homotopy through immersions to the unknot; see Definition [5.1. By Proposition 5.17, $E_{m}$ is homotopy equivalent to $\operatorname{Emb}\left(\mathbb{I}, \mathbb{I}^{m}\right) \times$ $\Omega \operatorname{Imm}\left(\mathbb{I}, \mathbb{I}^{m}\right)$. In [28, McClure and Smith define a cosimplicial object $\mathcal{O}^{\bullet}$ associated to an operad with multiplication $\mathcal{O}$, whose homotopy invariant totalization we denote $\widehat{\operatorname{Tot}}\left(\mathcal{O}^{\bullet}\right)$; see Definition 2.17 and Definition 2.5 below. Let $\mathcal{K}_{m}$ denote the $m$ th Kontsevich operad, introduced in 22, whose entries are compactified configuration spaces and which is weakly equivalent to the little $m$-disks operad 37; see Definition 4.1 and Theorem 4.5.

Theorem 1.1. The totalization of the Kontsevich operad $\widetilde{\operatorname{Tot}}\left(\mathcal{K}_{m}^{\bullet}\right)$ is homotopy equivalent to the inverse limit of the Taylor tower approximations for $E_{m}$ in the calculus of embeddings. Moreover, $\widetilde{\operatorname{Tot}}^{n}\left(\mathcal{K}_{m}^{\bullet}\right)$ is the nth degree approximation.

Building on work of Goodwillie, Klein and Weiss [46], 16, 20, [19, and Volic 43, 44, we have the following.

Corollary 1.2. For $m>3, E_{m}$ is weakly equivalent to $\widetilde{\operatorname{Tot}}\left(\mathcal{K}_{m}^{\bullet}\right)$. For $m=3$, all real-valued finite-type invariants of framed knots factor through a map from $E_{m}$ to $\widehat{\operatorname{Tot}}\left(\mathcal{K}_{m}^{\bullet}\right)$.

Applying the homology spectral sequence of a cosimplicial space, we have the following.

Corollary 1.3. For $m>3$, there is a spectral sequence with the $E^{2}$-term given by the Hochschild cohomology of the degree $m-1$ Poisson operad and which converges to the homology of $E_{m}$.

These results resolve conjectures of Kontsevich from his address at the AMS Mathematical Challenges Conference in the summer of 2000 23. Kontsevich's insights were motivated by novel combinatorial work of Tourtchine. In [40] Tourtchine gives an algebraic description of the $E^{1}$-term of Vassiliev's homology spectral sequence closely related to our Corollary 1.3 . Our results are at the level of spaces and show that the disagreement which Tourtchine found between the Hochschild

Received by the editors September 20, 2004.

2000 Mathematics Subject Classification. Primary 57Q45, 18D50, 57M27.

Key words and phrases. Knot spaces, operads, embedding calculus.

The author is partially supported by NSF grant DMS-0405922.

(C)2005 American Mathematical Society Reverts to public domain 28 years from publication 
cohomology of the Poisson operad and Vassiliev's $E^{2}$-term is accounted for by the fact that $E_{m}$ is not the classical knot space but is instead the space of knots with trivialization through immersions.

Our results bring together some recent developments in algebraic topology and its application to fields such as deformation theory and knot theory. In [36] we presented models for spaces of knots, including a cosimplical model which is analogous to the cosimplicial model for loop spaces. We build on these results in proving Theorem 1.1. In 28. McClure and Smith resolved the integral Deligne conjecture, showing that the totalization of an operad with multiplication has a two-cubes action both in the setting of chain complexes and that of spaces. We apply their results to establish the following.

Theorem 1.4. For any $m$, there is a little two-cubes action on $\widetilde{\operatorname{Tot}}\left(\mathcal{K}_{m}^{\bullet}\right)$. For $m>3, E_{m}$ is a two-fold loop space.

We conjecture that this two-cubes action on $\widetilde{\operatorname{Tot}}\left(\mathcal{K}_{m}^{\bullet}\right)$ is compatible with a twocubes action on the space of framed knots which has recently been defined by Budney [7, who goes on to show that long knots in dimension three are free over the two-cubes action. In future work we plan to investigate analogues of this freeness result in higher dimensions. A first step will be to construct operations compatible with the two-cubes structure in the homology spectral sequence for an operad with multiplication, as McClure and Smith currently plan to do. On the $E^{1}$-term such operations will presumably coincide with Tourtchine's bracket, defined combinatorially in [40, but through their space-level construction would also be compatible with differentials and extend to further terms.

Some of the technical results developed in this paper may be of independent interest. We fully develop the operad structure, with multiplication, on the simplicial compactification of configurations in Euclidean spaces. An operad structure on the canonical (Axelrod-Singer) compactification is known [14, 25] but does not yield an operad with multiplication. Instead there is a map from Stasheff's $A_{\infty}$ operad. Our approach to the operad structure on the simplicial variant blends geometry and combinatorics, revealing an operad structure on the standard simplicial model for the two-sphere.

\section{Contents}

1. Introduction

2. Background material

2.1. Cosimplicial spaces

2.2. Operads

3. The choose-two operad

4. The Kontsevich operad

5. Models for spaces of knots and immersions arising from the calculus of embeddings

5.1. A brief overview of the calculus of embeddings

5.2. Knot space models through homotopy limits of configuration spaces

5.3. A closer look at $E_{m}$

6. The main result

7. Observations and consequences 
7.1. Spectral sequences

7.2. A little two-cubes action from the McClure-Smith framework

Acknowledgements

References

\section{BACKGROUND MATERIAL}

Our main results are stated in terms of operads with multiplication and their associated cosimplicial spaces. As a chance to set the choice of definitions and notation which will be most convenient, and as an opportunity to place all standard material together, we review this material here. For a more complete survey we highly recommend [30. In particular, Section 3 of 30] introduces cosimplicial spaces, and Section 6 briefly introduces operads. The paper 26 gives a more comprehensive introduction to operads. A reader familiar with these constructions may wish to skip this section and refer back for clarification as needed.

\subsection{Cosimplicial spaces.}

Definition 2.1. Let $\Delta$ denote the category with one object for every non-negative integer and where the morphisms from $k$ to $\ell$ are the order-preserving maps from $[k]=\{0, \cdots, k\}$ to $[\ell]=\{0, \cdots, \ell\}$, ordered in the standard way. A cosimplicial object in a category $\mathcal{C}$ is a (covariant) functor from $\Delta$ to $\mathcal{C}$. A simplicial object is a contravariant functor from $\Delta$ to $\mathcal{C}$.

Cosimplicial objects are denoted $X^{\bullet}$, where $X^{k}$ is the image of $[k]$ under the functor, also known as the $k$ th entry. Simplicial objects are denoted $X_{\bullet}$. Central in the theory is the standard cosimplicial space $\Delta^{\bullet}$, whose $k$ th entry is $\Delta^{k}$, with vertices labelled by $[k]$, and which sends a morphism $[k] \rightarrow[\ell]$ to the linear map which extends this map on vertices.

Every order-preserving map $[k] \rightarrow[\ell]$ can be factored through elementary maps $d^{i}$, which are an isomorphism but for one element - namely $i$ - not in their image, and elementary maps $s^{i}$, which are an isomorphism but for having $i$ and $i+1$ in $[k]$ both mapping to $i \in[\ell]$. The corresponding maps between entries of simplicial and cosimplicial objects, called (co)face and (co)degeneracy maps, are often taken as a basis for their definition. The definitions are arranged so that the simplices of an ordered simplicial complex form a simplicial set. Indeed, a simplicial set or simplicial space $X_{\bullet}$ determines a space called its realization and denoted $\left|X_{\bullet}\right|$, defined as the quotient space of the union of $X_{i} \times \Delta^{i}$ over all $i$ by the relations $d_{j} x \times \beta \sim x \times d^{j} \beta$ and $s_{j} x \times \beta \sim x \times s^{j} \beta$ for all $x \in X^{i}, \beta \in \Delta^{i}$.

Cosimplicial spaces naturally arise when studying mapping spaces. The totalization of a cosimplicial space $\operatorname{Tot} X^{\bullet}$ is the space of natural transformations from $\Delta^{\bullet}$ to $X^{\bullet}$, which is first used tautologically to study mapping spaces as follows.

Definition 2.2. For any $X \in \mathcal{C}$, a category whose categorical product $\odot$ is symmetric monoidal, taking the product of $X$ with itself gives rise to a functor $X^{-}:$FSet $^{o p} \rightarrow \mathcal{C}$ which sends $S$ to $\bigodot_{s \in S} X$, where FSet is the category of finite sets. By composing a simplicial set $Y_{\bullet}: \Delta \rightarrow$ FSet $^{o p}$ with this functor, we obtain a cosimplicial object $X^{Y}$. 
Proposition 2.3. If $Y_{\bullet}$ is a simplicial set and $X$ is a space, $\operatorname{Tot}\left(X^{Y_{\bullet}}\right)$ is homeomorphic to the space of maps from $\left|Y_{\bullet}\right|$ to $X$.

For based $X$ and $Y_{\bullet}$ we may replace $X^{Y_{n}}$ by the subspace consisting of based maps from $Y_{n}$ to $X$. The resulting cosimplicial space, which we denote $X_{\star}^{Y \bullet}$, has totalization homeomorphic to the space of based maps from $\left|Y_{\bullet}\right|$ to $X$. Another interesting example along these lines is the Hochschild simplicial vector space $A^{S_{\bullet}^{1}}$, whose associated chain complex computes Hochschild homology of a commutative algebra $A$.

Cosimplicial spaces are intimately connected with homotopy limits (in fact, homotopy limits are defined in terms of cosimplicial spaces in [5]). The nerve of a category $C$ is the simplicial set $C_{\bullet}$, with $C_{i}$ being the collection of $i$ composable morphisms and structure maps defined through composing such maps or inserting identity maps (see, for example, Chapter 14 of [21]). Denote the realization of the nerve of $C$ by $B C$, also called the classifying space. Recall that if $c$ is an object of $C$, the category $C \downarrow c$ has objects which are maps with target $c$ and morphisms given by morphisms in $C$ which commute with these structure maps. The classifying space $B(C \downarrow c)$ is contractible because $C \downarrow c$ has a final object, namely $c$ mapping to itself by the identity morphism. A morphism $g$ from $c$ to $d$ induces a map from $C \downarrow c$ to $C \downarrow d$, so that $B(C \downarrow-)$ is itself a functor from $C$ to spaces.

Definition 2.4. The homotopy limit of a functor $E$ from a small category $C$ to the category of spaces is $\operatorname{Nat}(B(C \downarrow-), E)$, the space of natural transformations from $B(C \downarrow-)$ to $E$.

For $X^{\bullet}$ fibrant in the standard model structure on cosimplicial spaces, that is those which satisfy the matching condition 10.4.6 of [5], Theorem 11.4.4 of [5] states that $\operatorname{Tot}\left(X^{\bullet}\right) \simeq \operatorname{holim}_{\Delta} X^{\bullet}$, an equivalence needed for many applications. For cosimplicial spaces which do not necessarily satisfy the matching condition, we use an alternate definition of totalization for which this equivalence is a tautology.

Definition 2.5. $\quad$ Let $\widetilde{\Delta^{\bullet}}$ be the cosimplicial space whose $[k]$ th entry is $B(\Delta \downarrow[k])$ and whose structure maps are the standard induced maps.

- For a cosimplicial space $X^{\bullet}$ let $\widetilde{\operatorname{Tot}} X^{\bullet}$, called the homotopy invariant totalization, denote the space of natural transformations from $\widetilde{\Delta^{\bullet}}$ to $X^{\bullet}$.

- Let $\widetilde{\operatorname{Tot}}^{k} X^{\bullet}$ denote the space of natural transformations from the $k$ th coskeleton of $\widetilde{\Delta}$ to $X^{\bullet}$.

- Let $\Delta_{k}$ denote the full subcategory of $\Delta$ whose objects are $[i]$ for $i \leq k$. Let $i_{k}: \Delta_{k} \rightarrow \Delta$ be the inclusion functor.

In Section 15 of [29] the notation $\widetilde{\Delta}$ and Tot is used for any cofibrant replacement for $\Delta^{\bullet}$ and the corresponding totalization in the model structure on cosimplicial spaces where all objects are fibrant (in the usual model structure from [5], all objects are cofibrant). We choose one model of cofibrant replacement for definiteness.

Definition 2.6. Define $\widetilde{\operatorname{Tot}}\left(X^{\bullet}\right)$ to be the homotopy limit of $X^{\bullet}$ and $\widetilde{\operatorname{Tot}}^{k}\left(X^{\bullet}\right) \cong$ holim $\left(X^{\bullet} \circ i_{k}\right)$.

The cosimplicial category $\Delta$ is also intimately related to the category of subsets of a finite set. 
Definition 2.7. Let $P(k)$ be the category of all subsets of $[k]=\{0, \cdots, k\}$ where morphisms are defined by inclusion. Let $P_{0}(k)$ be the full subcategory of non-empty subsets.

The connection of this category to the simplicial world is evident in the identification of $B P_{0}(k)$ with the barycentric subdivision of a $k$-simplex. We use this identification to define maps $\widetilde{\Delta}^{k} \rightarrow \Delta^{k}$, and thus $\operatorname{Tot} X^{\bullet} \rightarrow \widetilde{\operatorname{Tot}} X^{\bullet}$ for any $X^{\bullet}$, through the identification of $\widetilde{\Delta}^{k}$ and $\Delta^{k}$ with $B(\Delta \downarrow[k])$ and $B P_{0}(k)$, respectively. Namely, take the map induced on classifying spaces by the functor which sends some $[n] \stackrel{f}{\rightarrow}[k]$ in $(\Delta \downarrow[k])$ to the image of $f$, as a subset of $[k]$. There is also a translation between cosimplicial diagrams and those indexed by $P_{0}(k)$, which we will use in Section 6 .

Definition 2.8. Let $c_{k}: P_{0}(k) \rightarrow \Delta$ be the functor which sends a subset $S$ to the object in $\Delta$ with the same cardinality, and which sends an inclusion $S \subseteq S^{\prime}$ to the composite $[i] \cong S \subset S^{\prime} \cong[j]$, where $[i]$ and $[j]$ are isomorphic to $S$ and $S^{\prime}$ respectively as ordered sets.

The following lemma is a consequence of Theorem 6.4 of [36], along with the observation that $\widetilde{\operatorname{Tot}}^{k}\left(X^{\bullet}\right)=\operatorname{holim}\left(i_{k} \circ X^{\bullet}\right)$.

Lemma 2.9. For $X^{\bullet}$ a cosimplicial space, holim $\left(X^{\bullet} \circ c_{k}\right)$ is weakly equivalent to $\widetilde{\operatorname{Tot}}^{k} X^{\bullet}$.

For a cosimplicial space there are spectral sequences for the homotopy groups [5] and homology groups [6], 35] of its (homotopy invariant) totalization, which we will apply in Section 7, The homotopy spectral sequence is straightforward, with convergence immediate from its definition through the tower

$$
\cdots \leftarrow \operatorname{Tot}^{i} X^{\bullet} \leftarrow \operatorname{Tot}^{i+1} X^{\bullet} \leftarrow \cdots,
$$

whose homotopy inverse limit is $\operatorname{Tot} X^{\bullet}$ and which is a tower of fibrations if $X^{\bullet}$ is fibrant. Unraveling the definitions we have the following.

Proposition 2.10. For a fibrant cosimplicial space $X^{\bullet}$ there is a spectral sequence converging to $\pi_{*}\left(\operatorname{Tot} X^{\bullet}\right)$ with $E_{-p, q}^{1}=\bigcap \operatorname{ker} s^{k}{ }_{*} \subseteq \pi_{q}\left(X^{p}\right)$. The $d_{1}$ differential is the restriction to this kernel of the map $\sum_{i=0}^{p+1}(-1)^{i} d^{i}{ }_{*}: \pi_{q}\left(X^{p-1}\right) \rightarrow \pi_{q}\left(X^{p}\right)$.

The homology spectral sequence is more subtle in its convergence. It generalizes the Eilenberg-Moore spectral sequence. One of the precise statements as to the convergence of this spectral sequence arising from [6] is as follows.

Theorem 2.11. For a fibrant cosimplicial space $X^{\bullet}$ there is a spectral sequence with $E_{-p, q}^{1}=\bigcap$ ker $s^{k}{ }_{*} \subseteq H_{q}\left(X^{p}\right)$. The $d_{1}$ differential is the restriction to this kernel of the map

$$
\sum_{i=0}^{p+1}(-1)^{i} d^{i}{ }_{*}: H_{q}\left(X^{p-1}\right) \rightarrow H_{q}\left(X^{p}\right) .
$$

This spectral sequence converges to $H_{*}\left(\operatorname{Tot} X^{\bullet}\right)$ if $X^{k}$ is simply connected for all $k$ and $E_{-p, q}^{1}=0$ when $q \geq$ cp for some $c>1$.

Alternately, one may arrive at the same spectral sequence from $E^{2}$ forward with $E_{-p, q}^{1}=H_{q}\left(X^{p}\right)$ and $d_{1}$ defined as before, but not restricted to the kernel of the codegeneracies. 
This theorem follows immediately from Theorem 3.2 of 6 and the Universal Coefficient Theorem, as both of Bousfield's conditions, namely that $E_{-p, q}^{1}=0$ if $p>q$ and that only finitely many $E_{-p, q}^{1}$ with $q-p=n$ are non-zero for any given $n$, follow from the vanishing with $q \geq c p$ for some $c>1$.

These spectral sequences apply unchanged to the homotopy invariant totalization, in which case the fibrancy condition can be dropped. If $X^{\bullet}$ is a cosimpicial space and $\underline{X^{\bullet}}$ is a fibrant replacement (as given by Proposition 8.1.3 and Theorem 15.3.4 in [21]), then

$$
\widetilde{\operatorname{Tot}}\left(X^{\bullet}\right)=\operatorname{Maps}\left(\widetilde{\Delta^{\bullet}}, X^{\bullet}\right) \simeq \operatorname{Maps}\left(\widetilde{\Delta^{\bullet}}, \underline{X^{\bullet}}\right) \simeq \operatorname{Maps}\left(\Delta^{\bullet}, \underline{X^{\bullet}}\right)=\operatorname{Tot}\left(\underline{X^{\bullet}}\right) .
$$

Because homotopy and homology of the entries and structure maps of $\underline{X^{\bullet}}$ agree with those of $X^{\bullet}$, the identifications of the $E^{1}$-terms of the associated spectral sequences are unchanged.

2.2. Operads. We define non- $\Sigma$ operads in terms of a well-known [3], 26], 36], 37] category of rooted trees.

Definition 2.12. - A rooted, planar tree (or rp-tree) is an isotopy class of finite connected acyclic graphs with a distinguished vertex called the root, embedded in the upper half-plane with the root at the origin so that the vertical coordinate in the plane is a monotone function which increases on each edge as the distance from the root increases. Univalent vertices of an rp-tree (not counting the root, if it is univalent) are called leaves.

- Each edge of the tree is oriented by the direction of the root path, which is the unique shortest path to the root. The vertex of an edge which is further from the root is called its inital vertex, and the vertex closer to the root is called its terminal vertex. We say that one vertex or edge lies over another if the latter is in the root path of the former. A non-root edge is called redundant if its initial vertex is bivalent.

- Given an rp-tree $T$ and a set of edges $E$ the contraction of $T$ by $E$ is the tree $T^{\prime}$ obtained by, for each edge $e \in E$, identifying its initial vertex with its terminal vertex (altering the embedding of the tree in a neighborhood of $e$ ) and removing $e$ from the set of edges.

- Let $\Upsilon$ denote the category of rp-trees, where there is a morphism from $T$ to $T^{\prime}$, denoted either $f_{T, T^{\prime}}$ or $c_{E}$, if $T^{\prime}$ is the contraction of $T$ along the set of non-leaf edges $E$.

- Both the collection of leaves in an rp-tree and the collections of edges with a given terminal vertex are ordered, using the clockwise orientation of the plane.

- A sub-tree of an rp-tree is a connected sub-graph. A sub-tree is an rp-tree through a linear isotopy which translates $v$ to the origin.

See Figure 2.14 for some examples of objects and morphisms in $\Upsilon$. Let $\Upsilon_{n}$ denote the full subcategory of trees with $n$ leaves. Note that $\Upsilon_{n}$ differs from $\Phi_{n}$ of [36], which is canonically isomorphic to the full subcategory of rp-trees without bivalent vertices. Each $\Upsilon_{n}$ has a terminal object, namely the unique tree with one vertex, called the $n$th corolla $\gamma_{n}$ as in [26]. We allow for the tree $\gamma_{0}$ which has no leaves, only a root vertex, and is the only element of $\Upsilon_{0}$. For a vertex $v$ let $|v|$ denote the number of edges for which $v$ is terminal, usually called the arity of $v$. 


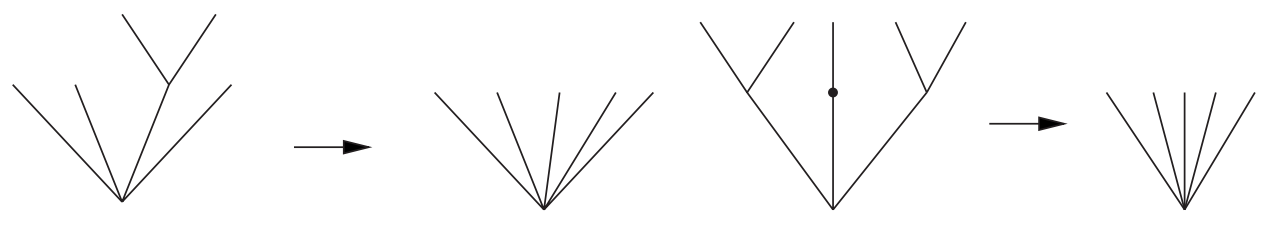

Figure 2.14. Two morphisms in $\Upsilon$ which give rise to standard operad structure maps. The first corresponds to a $\circ_{i}$ operation, the second to one of May's structure maps.

Definition 2.13. A non- $\Sigma$ operad is a functor $\mathcal{O}$ from $\Upsilon$ to a symmetric monoidal category $(\mathcal{C}, \odot)$ which satisfies the following axioms.

(1) $\mathcal{O}(T)=\odot_{v \in T} \mathcal{O}\left(\gamma_{|v|}\right)$.

(2) $\mathcal{O}\left(\gamma_{1}\right)=\mathbf{1}_{\mathcal{C}}=\mathcal{O}\left(\gamma_{0}\right)$.

(3) If $e$ is a redundant edge and $v$ is its terminal vertex, then $\mathcal{O}\left(c_{\{e\}}\right)$ is the identity map on $\odot_{v^{\prime} \neq v} F\left(\gamma_{v^{\prime}}\right)$ tensored with the isomorphism $\left(\mathbf{1}_{\mathcal{C}} \odot-\right)$ under the decomposition of axiom (11).

(4) If $S$ is a sub-tree of $T$ and if $f_{S, S^{\prime}}$ and $f_{T, T^{\prime}}$ contract the same set of edges, then under the decomposition of (10), $F\left(f_{T, T^{\prime}}\right)=F\left(f_{S, S^{\prime}}\right) \odot i d$.

We sketch the equivalence of this definition with two standard ones. By axiom (11), the values of $\mathcal{O}$ are determined by its values on the corollas $\mathcal{O}\left(\gamma_{n}\right)$, which correspond to $\mathcal{O}(n)$ in the usual operad terminology of [27]. Axioms (2) and (3) correspond to the unit condition. By axiom (4), the values of $\mathcal{O}$ on morphisms may be computed by composing morphisms on sub-trees, so we may identify some subset of basic morphisms through which all morphisms factor. In Figure 2.14 we illustrate some basic morphisms in $\Upsilon$. The first corresponds to what are known as $\circ_{i}$ operations. The second corresponds to May's operad structure maps from Definition 1.1 of [27. That $\mathcal{O}$ is a functor implies the commutativity of diagrams involving these basic morphisms. Another basic class extending these is that of all morphisms $T \rightarrow \gamma_{n}$ where $\gamma_{n}$ is a corolla and $T$ is any tree.

Example 2.15. $\quad$ - The associative operad $\mathcal{A}$, defined in any symmetric monoidal category, has $\mathcal{A}(T)=\mathbf{1}_{\mathcal{C}}$, and $\mathcal{A}\left(T \rightarrow T^{\prime}\right)=i d$ for all morphisms in $\Upsilon$.

- Let $\Phi$ denote the full subcategory of rp-trees with no redundant edges (called the category of reduced trees in [26]) and let $P: \Upsilon \rightarrow \Phi$ denote the functor which contracts all of the redundant edges of an rp-tree. The operad of planar trees, Tree $_{n}$ from Definition 1.41 of [26], is the operad in the category of sets which sends $T$ to the set of all $T^{\prime} \in \Phi$ which map to $P(T)$. It sends a contraction of edges of $T$ to the collection of contractions on the corresponding edges for trees over $P(T)$.

Definition 2.16. A map between non- $\Sigma$ operads is a natural transformation which respects the decomposition of axiom (11) of Definition 2.13. An operad with multiplication is a non- $\Sigma$ operad $\mathcal{O}$ equipped with a map from the associative operad $\mathcal{A}$.

The notion of operad with multiplication is due to Gerstenhaber and Voronov 13. The canonical example is the endomorphism operad of an associative algebra $\operatorname{End}(A)$. Algebras over an operad with multiplication are, in particular, associative 
algebras. An operad with multiplication in the category of spaces is an operad in the category of pointed spaces.

While we have taken a categorical approach to defining operads, we will take a more coordinatized approach to their associated cosimplicial objects. Recall the $\circ_{i}$ operations $\circ_{i}: \mathcal{O}(n) \odot \mathcal{O}(m) \rightarrow \mathcal{O}(n+m-1)$ which provide a basic set of morphisms for an operad, as illustrated in Figure 2.14. From section 3 of [28] we have the following.

Definition 2.17. - Given an operad with multiplication $\mathcal{O}$, let $\mu$ denote the morphism $\mathcal{A}(2)=\mathbf{1}_{\mathcal{C}} \rightarrow \mathcal{O}(2)$.

- Define $d^{i}: \mathcal{O}(n) \rightarrow \mathcal{O}(n+1)$ by

$$
d^{i}=\left\{\begin{array}{l}
\mathbf{1}_{\mathcal{C}} \odot \mathcal{O}(n) \stackrel{\mu \odot i d}{\rightarrow} \mathcal{O}(2) \odot \mathcal{O}(n) \stackrel{\circ_{1}}{\rightarrow} \mathcal{O}(n+1) \text { if } i=0 \\
\mathcal{O}(n) \odot \mathbf{1}_{\mathcal{C}} \stackrel{i d \odot \mu}{\longrightarrow} \mathcal{O}(n) \odot \mathcal{O}(2) \stackrel{\circ_{i}}{\rightarrow} \mathcal{O}(n+1) \text { if } 0<i<n+1 \\
\mathbf{1}_{\mathcal{C}} \odot \mathcal{O}(n) \stackrel{\mu \odot i d}{\rightarrow} \mathcal{O}(2) \odot \mathcal{O}(n) \stackrel{\circ_{2}}{\rightarrow} \mathcal{O}(n+1) \text { if } i=n+1 .
\end{array}\right.
$$

- Define $s^{i}$ as $\mathcal{O}\left(c_{i}\right)$ where $c_{i}: \gamma_{n} \rightarrow \gamma_{n-1}$ contracts the $i$ th leaf of $\gamma_{n}$.

- Let $\mathcal{O}^{\bullet}$ be the cosimplicial object in $\mathcal{C}$ whose $n$th entry is $\mathcal{O}(n)$ and whose coface and codegeneracy maps are given by $d^{i}$ and $s^{i}$ above. If $\mathcal{C}$ is the category of vector spaces over a given field, let $H H^{*}(\mathcal{O})$ be the homology of the cochain complex defined by the cosimplicial vector space $\mathcal{O}^{\bullet}$. If $\mathcal{C}$ is the category of spaces, we call $\widehat{\operatorname{Tot}}\left(\mathcal{O}^{\bullet}\right)$ the totalization of $\mathcal{O}^{\bullet}$.

It is straightforward to show that the maps $d^{i}$ and $s^{i}$ satisfy cosimplicial identities.

Remark. In the category of vector spaces, Tourtchine introduced the terminology $H H^{*}(\mathcal{O})$ because if $A$ denotes an associative algebra and $\operatorname{End}(A)$ is its endomorphism operad, then $H H^{*}(\operatorname{End}(A))=H H^{*}(A)$, the usual Hochschild cohomology of $A$. We are not aware, however, of any sense in which Hochschild cohomology of operads is a cohomology theory for operads. Instead, Kontsevich conjectures that there is a suitable enriched homotopy structure on the category of operads of spaces such that $\widetilde{\operatorname{Tot}}\left(\mathcal{O}^{\bullet}\right)$ is the derived space of maps from the associative operad to $\mathcal{O}$.

\section{The Choose-Two OPERAD}

At the combinatorial heart of our work is the choose-two operad. Its operad structure intertwines the sets $\left(\begin{array}{c}\mathbf{n} \\ 2\end{array}\right)$, of distinct pairs of elements $(i, j) \in \mathbf{n}=\{1, \ldots, n\}$ with $i<j$ for definiteness, with rooted planar trees. Recall that FSet ${ }^{o p}$, the opposite category to the category of pointed sets, is symmetric monoidal with product given by pointed union, denoted $\vee$, and unit given by the one-point set. Let $S_{+}$ denote the union of a set $S$ with a disjoint base point.

Definition 3.1. - The join of two leaves in an rp-tree is the first vertex (that is, the farthest from the root) at which their root paths coincide.

- Label both the leaves of an rp-tree and the edges which emanate from a given vertex $v$ with elements of $\mathbf{n}$ and $\{1, \ldots,|v|\}$ respectively according to the order given by planar embedding. To an rp-tree $T$ with $n$ leaves and two distinct integers $i, j \in \mathbf{n}$ let $v$ be the join of the leaves labelled $i$ and $j$ and define $J_{v}(i), J_{v}(j)$ to be the labels of the edges of $v$ over which leaves $i$ and $j$ lie, as illustrated in Figure 3.2. 

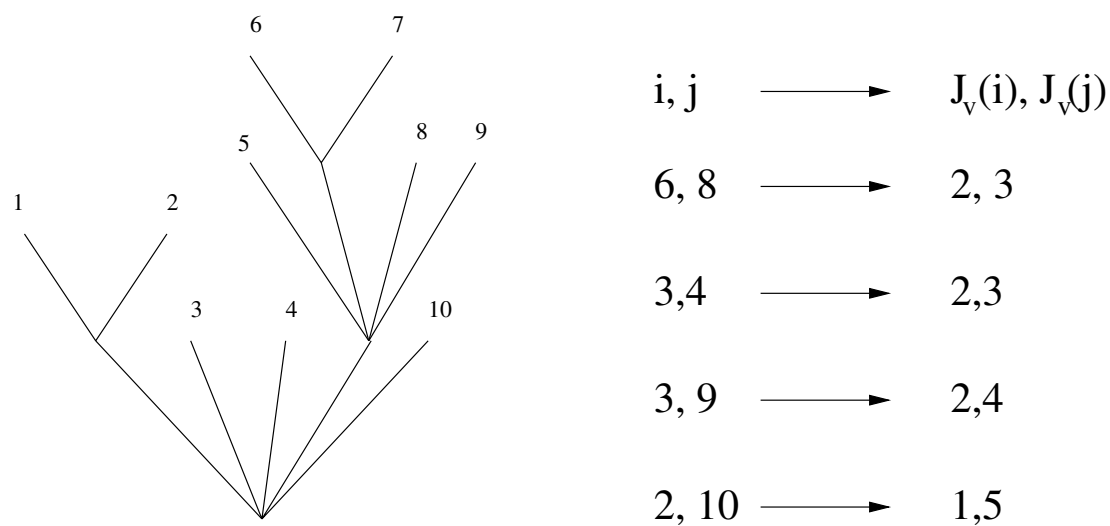

FiguRE 3.2.

- Let $\mathcal{B}$, the choose-two operad, be the non- $\Sigma$ operad in the category FSet ${ }^{o p}$ defined as follows:

$-\mathcal{B}(T)=\bigvee_{w}\left(\begin{array}{c}|w| \\ 2\end{array}\right)_{+}$, where $w$ ranges over vertices of $T$;

$-\mathcal{B}\left(T \rightarrow \gamma_{n}\right)$, where $\gamma_{n}$ is a corolla, is the function

$$
(i, j) \in\left(\begin{array}{c}
\mathbf{n} \\
2
\end{array}\right) \mapsto\left(J_{v}(i), J_{v}(j)\right) \in\left(\begin{array}{c}
|v| \\
2
\end{array}\right) \subset \bigvee_{w \in T}\left(\begin{array}{c}
|w| \\
2
\end{array}\right)_{+},
$$

where $v$ is the join of leaves $i$ and $j$.

With our choice of definitions, it is straightforward to verify that $\mathcal{B}$ is an operad.

Theorem 3.3. As a simplicial set, the cosimplicial object in FSet ${ }^{o p}$ associated to the choose-two operad $\mathcal{B}^{\bullet}$ is isomorphic to $S_{\bullet}^{2}$, the simplicial model for $S^{2}$.

Proof. Recall that $S_{\bullet}^{2}=\Delta_{\bullet}^{2} / \partial \Delta_{\bullet}^{2}$, where $\Delta_{\bullet}^{2}$ is the standard simplicial model for $\Delta^{2}$. The set $n$-simplices of $\Delta^{2}$ is the set of $\left(x_{0} \leq x_{1} \leq \cdots \leq x_{n}\right) \in\{0,1,2\}^{n+1}$, so the cardinality of $\Delta_{n}^{2}$ is the $(n+1)$ st triangular number. The $i$ th face and degeneracy maps are defined by deleting and repeating $x_{i}$, respectively. To obtain $S_{\bullet}^{2}$ we identify all $n$-tuples in which one of $\{0,1,2\}$ does not appear to a single simplex in each degree, which is degenerate in positive degrees.

The $n$th entry of $S_{\bullet}^{2}$ is isomorphic to $\left(\begin{array}{l}\mathbf{n} \\ 2\end{array}\right)_{+}$, the set of unordered pairs of points in $\mathbf{n}$, along with a disjoint point + which is the image of $\partial \Delta_{\text {. }}^{2}$ under the quotient map. The isomorphism records the indices $j$ and $k$ for which $x_{j-1}<x_{j}$ and $x_{k-1}<x_{k}$, when there are two such indices. When there are not two such indices, such a sequence is identified with the degenerate point + . Under this isomorphism $d_{i}$ sends + to + and for $i \neq 0, n$ sends

$$
(j, k) \mapsto\left\{\begin{array}{l}
\left(\delta_{i}(j), \delta_{i}(k)\right) \text { if } \delta_{i}(j) \neq \delta_{i}(k) \quad \text { where } \delta_{i}(j)=\left\{\begin{array}{l}
j \text { if } j \leq i \\
+ \text { otherwise }
\end{array}-1 \text { if } j>i .\right.
\end{array}\right.
$$

For $i=0$ and $i=n$ the basic formula is the same, but the $(j, k)$ which get sent to + are those with $j=1$ or $k=n$, respectively. Similarly, $s_{i}$ sends + to + and sends $(j, k) \mapsto\left(\sigma_{i}(j), \sigma_{i}(k)\right)$ where $\sigma_{i}(j)=j$ if $j \leq i$ or $j+1$ otherwise. 
By definition $\mathcal{B}^{n}=\left(\begin{array}{l}\mathbf{n} \\ 2\end{array}\right)_{+}$, and it is straightforward to check that the structure maps of $S_{\bullet}^{2}$ and the associated cosimplicial object of $\mathcal{B}^{\bullet}$ coincide. To give an example, we unravel the definition of $d^{i}$ with $0<i<n$ for $\mathcal{B}^{n}$. These coface maps are given by composites $\left(\mathcal{B}\left(\gamma_{n}\right) \vee+\right) \stackrel{i d \vee \mu}{\longrightarrow} \mathcal{B}\left(\Downarrow_{n}^{i}\right) \stackrel{\circ_{i}}{\longrightarrow} \mathcal{B}\left(\gamma_{n+1}\right)$, where $\Downarrow_{n}^{i}$ denotes the tree with $n$ root edges and one trivalent internal vertex, which is terminal for the $i$ th root edge. In FSet ${ }^{o p}$ the morphism $i d \vee \mu$ corresponds to the collapse map in FSet which sends $\left(\begin{array}{l}\mathbf{2} \\ 2\end{array}\right)_{+} \subset\left(\begin{array}{l}\mathbf{n} \\ 2\end{array}\right)_{+} \vee\left(\begin{array}{l}\mathbf{2} \\ 2\end{array}\right)_{+}$to the base point and is the identity on $\left(\begin{array}{l}\mathbf{n} \\ 2\end{array}\right)_{+}$. The morphism $o_{i}$ sends $(i, i+1)$ to $(1,2) \in\left(\begin{array}{l}\mathbf{2} \\ 2\end{array}\right)$ and sends all other $(j, k)$ to $\left(\delta_{i}(j), \delta_{i}(k)\right) \in\left(\begin{array}{c}\mathbf{n} \\ 2\end{array}\right)$. The composite of these two maps coincides with the definition of $d_{i}$ for $S_{\bullet}^{2}$, as in Equation (1).

Let $X^{\mathcal{B}^{\bullet}}$ be the operad which is the composite of the operad $\mathcal{B}^{\bullet}: \Upsilon \rightarrow$ FSet $^{\text {op }}$ with the symmetric monoidal functor $X^{-}:$FSet $^{o p} \rightarrow$ Top. Theorem 3.3 implies the following.

Corollary 3.4. For any $X$ in a category $\mathcal{C}$ whose categorical product is symmetric monoidal, $X^{S^{2}}$ canonically defines an operad through its isomorphism with $X^{\mathcal{B}^{\bullet}}$.

We have yet to find any familiar interpretation for algebras over this operad in the categories of spaces and vector spaces. For spaces the operad structure on $X^{S_{\bullet}^{2}}$ does have consequences, as we explain in Example 7.4 .

\section{The Kontsevich OPERAD}

In this section we define an operad structure on the completion of configurations in Euclidean space up to translation and scaling defined by Kontsevich 22. The fact that one could define operads using the canonical completion of configuration spaces was noticed by Getzler and Jones 14 soon after this completion was introduced by Fulton-MacPherson 11 and Axelrod-Singer 11. This operad structure was fully developed by Markl [25. The variant with which we work was first proposed by Kontsevich [22, but Gaiffi [12] pointed out the deviation with the canonical completion. Indeed, while Kontsevich called the following the Fulton-MacPherson operad, we call it the Kontsevich operad to highlight the difference between the two. Though this construction lacks some of the properties of the canonical completion, in particular smoothness, it has diagonal and projection maps which satisfy simplicial identities exactly rather than up to homotopy. These properties led to this construction's independent discovery, its use, and its naming as the simplicial variant in 36 .

We start by setting notation for products of spaces and maps, which we will use extensively.

Notation. If $S$ is a finite set, let $X^{S}$ be the space of all functions from $S$ to $X$ with the product topology. For coordinates we use $\left(x_{s}\right)_{s \in S}$ or just $\left(x_{s}\right)$ when $S$ is understood. Similarly, a product of maps $\prod_{s \in S} f_{s}$ may be written $\left(f_{s}\right)_{s \in S}$ or just $\left(f_{s}\right)$. Recall that $\mathbf{n}=\{1, \ldots, n\}$.

Definition 4.1. - Let $C_{n}\left(\mathbb{R}^{m}\right)$ denote the space of $\left(x_{i}\right) \in\left(\mathbb{R}^{m}\right)^{\mathbf{n}}$ such that if $i \neq j$, then $x_{i} \neq x_{j}$. Let $\widetilde{C}_{n}\left(\mathbb{R}^{m}\right)$ be the quotient of $C_{n}\left(\mathbb{R}^{m}\right)$ by the equivalence relation generated by translating all of the $x_{i}$ by some $v$ or multiplying them all by the same positive scalar.

- For any $v \in \mathbb{R}^{m}-0$, let $u(v)=\frac{v}{\|v\|}$, the unit vector in the direction of $v$. 
- Let $\widetilde{C}_{n}\left\langle\left[\mathbb{R}^{m}\right]\right\rangle$ be the closure of the image of $\widetilde{C}_{n}\left(\mathbb{R}^{m}\right)$ under the map $\left(\pi_{i j}\right)$ to $\left(S^{m-1}\right)^{\left(\begin{array}{c}\mathrm{n} \\ 2\end{array}\right)}$, where $\pi_{i j}$ sends the equivalence class of $\left(x_{i}\right)$ to $u\left(x_{i}-x_{j}\right)$.

Note that $\left(\pi_{i j}\right)$ is not injective - it fails to be so on configurations in which all the $x_{i}$ lie on some line - so $\widetilde{C}_{n}\left(\mathbb{R}^{m}\right)$ is not a subspace of $\widetilde{C}_{n}\left\langle\left[\mathbb{R}^{m}\right]\right\rangle$. But we do have the following theorem, a consequence of Corollary 4.5, Lemma 4.12 and Corollary 5.10 of [37].

Theorem 4.2. The canonical map $\widetilde{C}_{n}\left(\mathbb{R}^{m}\right) \rightarrow \widetilde{C}_{n}\left\langle\left[\mathbb{R}^{m}\right]\right\rangle$ is a homotopy equivalence.

What makes $\widetilde{C}_{n}\left\langle\left[\mathbb{R}^{m}\right]\right\rangle$ manageable is that we can characterize it as a subspace of $\left(S^{m-1}\right)^{\left(\begin{array}{l}\mathbf{n} \\ 2\end{array}\right)}$. We extend coordinates for $\left(u_{i j}\right) \in\left(S^{m-1}\right)^{\left(\begin{array}{l}\mathbf{n} \\ 2\end{array}\right)}$ by letting $u_{j i}$ be $-u_{i j}$ when $j>i$.

Definition 4.3. - A chain, or $k$-chain, in $S$ is a collection $\left\{i_{1} i_{2}, i_{2} i_{3}, \ldots\right.$, $\left.i_{k-1} i_{k}\right\}$, with all $i_{j} \in S$ and $i_{j} \neq i_{j+1}$. Such indices label the edges of a path in the complete graph on $S$. A chain is a loop, or $k$-loop, if $i_{k}=i_{1}$. A chain is straight if it does not contain any loops. The reversal of a chain is the chain $i_{k} i_{k-1}, \ldots, i_{2} i_{1}$.

- A point $\left(u_{i j}\right) \in\left(S^{m-1}\right)^{\left(\begin{array}{l}\mathbf{n} \\ 2\end{array}\right)}$ is three-dependent if for any 3-loop $L$ in $\mathbf{n}$ there exist $a_{i j} \geq 0$, with at least one non-zero, such that $\sum_{i j \in L} a_{i j} u_{i j}=0$.

- If $S$ has cardinality four and is ordered, we may associate to a straight 3chain $C$ a permutation of $S$ denoted $\sigma(C)$ which relates the order in which indices appear in $C$ to the ordering on $S$. For example, if $S=\{1,2,3,4\}$, then $\sigma(23,31,14)=(2314)$. A complementary 3 -chain $C^{*}$ is a chain, unique up to reversal, which comprises the three pairs of indices not in $C$.

- A point $\left(u_{i j}\right) \in\left(S^{m-1}\right)^{\left(\begin{array}{c}\mathbf{n} \\ 2\end{array}\right)}$ is four-consistent if for any $S \subset \mathbf{n}$ of cardinality four and any $v, w \in S^{m-1}$ we have that

$$
\sum_{C \in \mathcal{C}^{3}(S)}(-1)^{|\sigma(C)|}\left(\prod_{i j \in C} u_{i j} \cdot v\right)\left(\prod_{i j \in C^{*}} u_{i j} \cdot w\right)=0
$$

where $\mathcal{C}^{3} S$ is the set of straight 3-chains in $S$ modulo reversal and $|\sigma(C)|$ is the parity of $\sigma(C)$.

Points in the image of $C_{n}\left(\mathbb{R}^{m}\right)$ under $\left(\pi_{i j}\right)$ are three-dependent and four-consistent, and also satisfy $u_{i j}=-u_{j i}$, a condition we refer to as anti-symmetry. These properties also hold for $C_{n}\left\langle\left[\mathbb{R}^{m}\right]\right\rangle$, the closure, by continuity. Adding the converse, we have the following, which is Theorem 5.14 of [37].

Theorem 4.4. $\widetilde{C}_{n}\left\langle\left[\mathbb{R}^{m}\right]\right\rangle$ is the subspace of all three-dependent, four-consistent points in $\left(S^{m-1}\right)^{\left(\begin{array}{l}\mathrm{n} \\ 2\end{array}\right)}$.

We will define operad maps on the completions $\widetilde{C}_{n}\left\langle\left[\mathbb{R}^{m}\right]\right\rangle$ through coordinates of $\left(S^{m-1}\right)^{\left(\begin{array}{c}\mathrm{n} \\ 2\end{array}\right)}$. Embed $\left(S^{m-1}\right)^{\left(\begin{array}{c}\mathrm{n} \\ 2\end{array}\right)}$, and thus $\widetilde{C}_{n}\left\langle\left[\mathbb{R}^{m}\right]\right\rangle$, in $\left(S^{m-1}\right)^{\left(\begin{array}{c}\mathrm{n} \\ 2\end{array}\right)}+$ as the subspace of $\left(u_{i j}\right) \times u_{+}$with $u_{+}$equal to the basepoint of $S^{m-1}$, which we choose to be the south pole $*_{S}=(0, \ldots, 0,-1)$.

Theorem 4.5. The operad structure on $\left(S^{m-1}\right)^{\mathcal{B}^{\bullet}}$ restricts to the subspaces $\widetilde{C}_{n}\left\langle\left[\mathbb{R}^{m}\right]\right\rangle$. 
We call the resulting operad, whose $n$th entry is $\widetilde{C}_{n}\left\langle\left[\mathbb{R}^{m}\right]\right\rangle$, the Kontsevich operad $\mathcal{K}_{m}$.

Proof. Given a tree $T$, let $\left(u_{k \ell}^{v}\right)$ be a point in $\left(S^{m-1}\right)^{\mathcal{B}(T)}$, where $v$ ranges over vertices of $T$ and $k, \ell \in\left(\begin{array}{c}|\mathbf{v}| \\ 2\end{array}\right)$. By Definition 3.1, the operad structure on $\left(S^{m-1}\right)^{\mathcal{B}^{\bullet}}$ sends the morphism $T \rightarrow \gamma_{n}$ to the map given in coordinates by $\left(u_{k \ell}^{v}\right) \mapsto\left(w_{i j}\right)_{i, j \in\left(\begin{array}{c}\mathbf{n} \\ 2\end{array}\right)}$, where $w_{i j}=u_{J_{v}(i), J_{v}(j)}^{v}$ and $v$ is the join vertex of the leaves $i$ and $j$.

We verify that if the $\left(u_{k \ell}^{v}\right)$ satisfy three-dependence and four-consistency for each $v$, then so does $\left(w_{i j}\right)$. For three-dependence, given some $w_{i j}, w_{j k}$ and $w_{k i}$, there are two cases to consider. In the first case the join in $T$ of leaves $i$ and $j$ lies over that of $i$ and $k$, so that $w_{j k}=-w_{k i}$ or $0 w_{i j}+1 w_{j k}+1 w_{k i}=0$. In the second case the joins of $i$ and $j$ and $k$ are all equal to the same $v$, so that the dependence of $w_{i j}, w_{j k}$ and $w_{k i}$ follows from that of $\left(u_{J_{v}(i) J_{v}(j)}^{v}\right),\left(u_{J_{v}(j) J_{v}(k)}^{v}\right)$ and $\left(u_{J_{v}(k) J_{v}(i)}^{v}\right)$. Four-consistency works similarly. Given indices $i, j, k$ and $\ell$ the pairwise joins could all equal some $v$, in which case four consistency of these $\left\{w_{i j}\right\}$ follows from that of $\left\{u_{k \ell}^{v}\right\}$. Or, if for example the join of $i$ and $j$ lies over those of $i, k$ and $\ell$, then $w_{i k}=w_{j k}$ and $w_{i \ell}=w_{j \ell}$, so four-consistency will follow by the canceling of terms which agree but for opposite signs.

In [37, we stratify $\widetilde{C}_{n}\left\langle\left[\mathbb{R}^{m}\right]\right\rangle$, and in particular the points added in closure. We will not need this stratification explicitly for our applications, but the related geometry is helpful in understanding the operad structure of $\mathcal{K}_{m}$. The stratification is indexed by rp-trees with no redundant edges, with the $T$ th stratum being the image of a map from $\prod_{v \in V(T)} \widetilde{C_{|v|}}\left(\mathbb{R}^{m}\right)$ to $\widetilde{C}_{n}\left\langle\left[\mathbb{R}^{m}\right]\right\rangle$ sending $\left(x_{i}^{v}\right) \mapsto\left(u_{i j}\right)$ with $u_{i j}=\pi_{J_{v}(i) J_{v}(j)}\left(\left(x_{i}\right)_{v}\right)$. Studying this stratification led us to the definition of the choose-two operad. See Section 3 and Theorem 5.14 of [37] for a full development of this geometry, which is illustrated in Figure 4.6 .

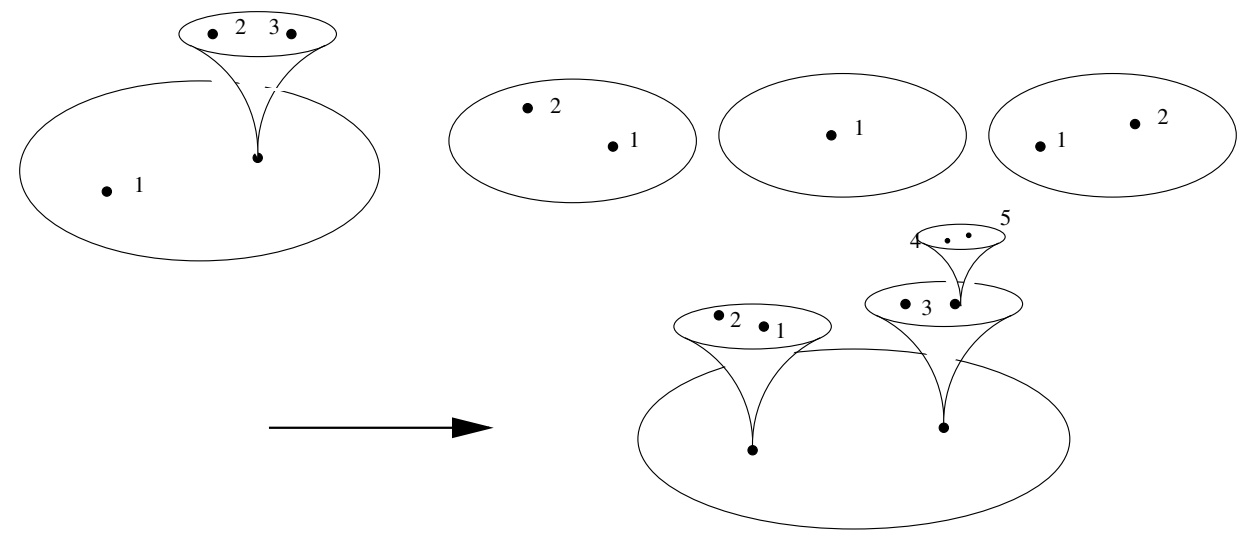

Figure 4.6. The effect of an operad structure map associated to the morphism $\forall Y-W$.

The standard completions $\widetilde{C_{n}}\left[\mathbb{R}^{m}\right]$ also constitute entries of an operad, which has been more intensively studied [14, 22], 25]. The reason we use $\mathcal{K}^{m}$ is the following. 
Proposition 4.7. The associative operad maps to the Kontsevich operad, for definiteness by choosing the basepoint $\left(x_{i j}\right) \in \widetilde{C_{n}}\left\langle\left[\mathbb{R}^{m}\right]\right\rangle$ with all $x_{i j}=*_{S}$, for all $n$.

Finally, we give a comparison between the little disks operad, which we need to formalize, and the Kontsevich operad.

Definition 4.8. - Recall that the space of $n$ little disks in $D^{m}$, the unit disk, denoted $D^{m}(n)$ is the subspace of $C_{n}\left(D^{m}\right) \times(0,1]^{n}$ of $\left(x_{i}\right) \times\left(r_{i}\right)$ such that the balls $B\left(x_{i}, r_{i}\right)$ are contained in $D^{m}$ and have disjoint interiors.

- Let $T$ be a tree whose vertices consist of the root vertex $v_{0}$ and a terminal vertex $v_{e}$ for each root edge $e$. Thus, $T \rightarrow \gamma_{n}$, where $n$ is the number of leaves of $T$, gives rise to one of May's structure maps as in Figure 2.14. Given a label $i \in \mathbf{n}$ let $v(i)$ be the initial vertex for the $i$ th leaf, let $o(i)$ be the label of leaf $i$ within the ordering on edges of $v(i)$ and let $e(i)$ be the label of the root edge for which $v(i)$ is terminal.

- Define $\mathcal{D}^{m}\left(T \rightarrow \gamma_{n}\right)$ as follows:

$\left(x_{i}^{v}, r_{i}^{v}\right)_{1 \leq i \leq \# v}^{v \in V(T)} \mapsto\left(y_{j}, \rho_{j}\right)_{j \in \mathbf{n}} \quad$ where $\quad y_{j}=x_{e(j)}^{v_{0}}+r_{e(j)}^{v_{0}} x_{o(j)}^{v(j)}$ and $\rho_{j}=r_{e(j)}^{v_{0}} r_{o(j)}^{v(j)}$.

Boardman and Vogt [4 and May [27] showed that connected algebras over $\mathcal{D}^{m}$ are $m$-fold loop spaces.

Theorem 4.9. Let $T$ be a tree with a vertex over each root edge as in Definition 4.8 above. The following diagram commutes up to homotopy,

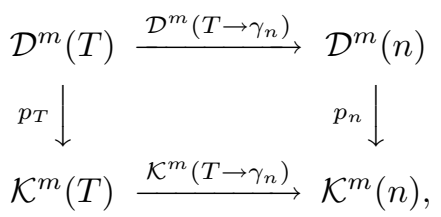

where the vertical maps $p_{T}$ are the products of projections $p_{n}: D^{m}(n) \rightarrow C_{n}\left(\mathbb{R}^{m}\right)$ composed with the canonical maps $C_{n}\left(\mathbb{R}^{m}\right) \rightarrow \widetilde{C}_{n}\left(\mathbb{R}^{m}\right) \rightarrow \widetilde{C}_{n}\left\langle\left[\mathbb{R}^{m}\right]\right\rangle$. Moreover, the vertical maps are homotopy equivalences.

Proof. We define the homotopy explicitly. Define $H: \mathcal{D}^{m}(T) \times(0,1] \rightarrow \widetilde{C}_{n}\left(\mathbb{R}^{m}\right)$ by sending $\left(x_{i}^{v}, r_{i}^{v}\right)$ as in Definition 4.8 and $t \in(0,1]$ to the equivalence class of $\left(y_{j}(t)\right)$ with $y_{j}(t)=x_{e(j)}^{v_{0}}+t \cdot r_{e(j)}^{v_{0}} x_{o(j)}^{v(j)}$. We claim that $H$ extends uniquely to $\bar{H}: \mathcal{D}^{m}(T) \times[0,1] \rightarrow \widetilde{C}_{n}\left\langle\left[\mathbb{R}^{m}\right]\right\rangle$, and that $\bar{H}$ coincides with $\mathcal{K}^{m}\left(T \rightarrow \gamma_{n}\right) \circ p_{T}$ when $t=1$. Consider $u_{i j}=\pi_{i j}\left(\left(y_{k}(t)\right)\right)$. If the join of leaves $i$ and $j$ is one of the non-root vertices, so $v(i)=v(j)$, then $u_{i j}$ will be equal to the unit vector in the direction of $x_{o(j)}^{v(j)}-x_{o(i)}^{v(j)}$, independent of $t$. If the join of leaves $i$ and $j$ is the root vertex, then as $t$ approaches $0, u_{i j}$ approaches the unit vector in the direction of $x_{e(j)}^{v_{0}}-x_{e(i)}^{v_{0}}$. These limiting values coincide with the definition of $\mathcal{K}^{m}\left(T \rightarrow \gamma_{n}\right)\left(x_{i}^{v}\right)$.

That the projection $D^{m}(n) \rightarrow C_{n}\left(D^{m}\right)$ is a homotopy equivalence is standard, known since the definition of little cubes in 3 , so by Theorem 4.2 the maps $p_{n}$ are homotopy equivalences.

In fact, 22] claims that these $\mathcal{D}^{m}$ and $\mathcal{K}^{m}$ are homotopy equivalent operads, which we assume to mean that there is a chain of equivalences of maps of operads, that is, maps which commute with structure maps exactly. We will not need this 
stronger claim. Recall that the homology of an operad of spaces with field coefficients is an operad of vector spaces by the Künneth theorem. The homology of the little disks operad has a well-known description.

Definition 4.10. The $k$ th entry of the degree $n$ Poisson operad $\operatorname{Poiss}_{n}(k)$ is the submodule of the symmetric algebra on the free graded Lie algebra over $k$ variables $x_{1}, \ldots, x_{k}$ spanned by monomials in which all variables appear exactly once. Monomials are graded by putting all $x_{i}$ in degree zero and giving the bracket degree $n$. So for example $\left[x_{1}, x_{3}\right]\left[\left[x_{4}, x_{2}\right] x_{5}\right]$ and $x_{1} x_{2} \cdots x_{5}$ are elements of Poiss ${ }_{3}(5)$ of degree nine and zero respectively.

The map $\circ_{i}: \operatorname{Poiss}_{n}(j) \otimes \operatorname{Poiss}_{n}(k) \rightarrow \operatorname{Poiss}_{n}(j+k-1)$ sends $m_{1} \otimes m_{2}$ to the monomial defined as follows.

- For each $j$, substitute $x_{j+i-1}$ for $x_{j}$ in $m_{2}$ to obtain $\overline{m_{2}}$.

- In $m_{1}$, substitute $x_{j+i-1}$ for $x_{j}$ if $j>i$ and $\overline{m_{2}}$ for $x_{i}$ to obtain $m$.

- Reduce $m$ according to the graded Leibniz rule

$$
[a, b c]=[a, b] c+(-1)^{(|a|+n+1)|b|} b[a, c],
$$

to obtain an element of $\operatorname{Poiss}_{n}(i+j-1)$.

The following corollary is essentially a summary of Fred Cohen's famous calculation of the homology of $\mathcal{D}^{m}$ [10. We also plan to give an exposition of this result in 38 .

Theorem 4.11. The homology of $\mathcal{K}^{m}$ is Poiss $_{m-1}$.

\section{MODELS FOR SPACES OF KNOTS AND IMMERSIONS ARISING FROM THE CALCULUS OF EMBEDDINGS}

5.1. A brief overview of the calculus of embeddings. Our main theorems connect the theory of operads to Goodwillie calculus. We first informally introduce some terminology from the calculus of embeddings (see Weiss [45] for an excellent introduction and [46] for a full treatment), and then precisely state the theorems we use. The main spaces with which we are concerned are related to embeddings and immersions.

Definition 5.1. Let $\operatorname{Emb}(M, N)$ denote the space of embeddings of $M$ in $N$, topologized as a subspace of the space of $C^{1}$ maps. Similarly, let $\operatorname{Imm}(M, N)$ be the space of immersions of $M$ in $N$. If $M$ and $N$ have boundaries, we usually specify some boundary conditions. In particular, if $M=\mathbb{I}$ and $N=\mathbb{I}^{m}$, we let $*_{+}=(0, \ldots, 0,1) \in \mathbb{I}^{m}, *_{-}=(0, \ldots, 0,-1)$ and demand that the endpoints of $\mathbb{I}$ map to $*_{+}$and $*_{-}$with tangent vectors $*_{S}$.

By results of Palais [32, these spaces are dominated by simplicial complexes and thus are homotopy equivalent to CW-complexes [31.

In the calculus of embeddings, we view spaces of embeddings, immersions and other moduli in differential topology as functors from the poset of open subsets of $M$ to topological spaces, a philosophy originally due to Gromov. Ultimately interested in the value of the functor at the open set which is all of $M$, we try to use homotopy limits to interpolate that value from values of the functor at simple open sets, namely those which are diffeomorphic to a union of finitely many disjoint open balls. Functors for which interpolation using a finite number of balls works perfectly are called polynomial, and those for which interpolation works in the limit 
as the number of balls tends to infinity are called analytic. Weiss shows in [46] that polynomial functors are those which satisfy higher-order Mayer-Vietoris conditions, and Goodwillie-Weiss show in [16] that analyticity follows from satisfying those conditions through an increasing range of connectivity. More formally we have the following.

Definition 5.2. $\quad$ - For any manifold $W$ of dimension $m$ let $\mathcal{U}(M)$ be the category of open subsets of $M$ under inclusion, and let $\mathcal{U}_{k}(M)$ be the full sub-category of $\mathcal{U}(M)$ of open sets diffeomorphic to $\bigsqcup_{i} \mathbb{R}^{m}$, where $i \leq k$.

- For any contravariant functor $F$ from $\mathcal{U}(M)$ to spaces let $T_{k} F$ be the functor which sends $W$ to $\operatorname{holim}_{U \in \mathcal{U}_{k}(W)} F(U)$.

- Let $b_{k}(F): T_{k} F \rightarrow T_{k-1} F$ be the canonical natural transformation defined by restricting $\mathcal{U}_{k}(M)$ to $\mathcal{U}_{k-1}(M)$, and let $T_{\infty} F$ be the homotopy inverse limit of the $T_{k} F$ over these restrictions.

- Let $\eta_{k}(F): F \rightarrow T_{k} F$ be the canonical natural transformation arising from the maps $F(M) \rightarrow F(U)$ for $U \in \mathcal{U}_{k}(M)$. If by context $F$ is understood, we will use the simpler notation $\eta_{k}$.

- The natural transformations $\eta_{k}$ commute with the $b_{k}$, so let $\eta_{\infty}: F \rightarrow T_{\infty} F$ be the limiting natural transformation.

The sequence $T_{0} F \stackrel{b_{1}}{\leftarrow} T_{1} F \stackrel{b_{2}}{\leftarrow} T_{2} F \leftarrow \cdots$ is called the Taylor tower for $F$. Analyticity means that the homotopy inverse limit of this tower is weakly equivalent to $F$. The motivating example for this circle of ideas is that of immersions.

Theorem 5.3. If $\operatorname{dim}(M)<\operatorname{dim}(N)$, then for $k \geq 1, \eta_{k}: \operatorname{Imm}(U, N) \rightarrow$ $T_{k} \operatorname{Imm}(U, N)$ is a weak equivalence for any $U \subseteq M$.

This theorem follows from Example 2.3 of [46, which says that $\operatorname{Imm}(-, N)$ is a linear functor, and the commentary after Theorem 5.1 of 46 . See also Theorem 5.15 below and Proposition 5.12 of 43 . The embedding functor is not polynomial but by theorems of Goodwillie, Klein and Weiss it is analytic. The following theorem is essentially Corollary 2.5 of [16].

Theorem 5.4. If $\operatorname{dim}(M)<\operatorname{dim}(N)-2$, then $\eta_{\infty}(\mathrm{Emb})$ is a weak equivalence.

For $\operatorname{dim}(M)<\operatorname{dim}(N)-2$ as stated, this theorem requires deep disjunction results of Goodwillie, and surgery results of Goodwillie-Klein [20, [19. If $\operatorname{dim}(M)$ is less than roughly $\frac{\operatorname{dim}(N)}{2}$, in particular when $M$ is one-dimensional and $N$ has dimension five or greater, there are much easier methods, using only the generalized Blakers-Massey theorem and dimension counting, for proving the needed higherorder Mayer-Vietoris conditions.

5.2. Knot space models through homotopy limits of configuration spaces. Definition 5.2 of $T_{k} \operatorname{Emb}(M, N)$ seems difficult to manage, being a homotopy limit over a large indexing category. But the building blocks, namely spaces of embeddings of balls, are essentially configuration spaces. Goodwillie, Klein and Weiss have used this observation to give more concrete models for the spaces in this Taylor tower (or for the homotopy fibers of $T_{k} \rightarrow T_{k-1}$, which are called layers), either as spaces of sections, as in section nine of [46, or as mapping spaces with strongly defined equivariance properties, as in [17. In the case of knots we have developed three closely-related models for these polynomial approximations 36] and used them for both computational and geometric applications [34, 9. These 
models all utilize completions of configuration spaces constructed similarly to the Kontsevich operad.

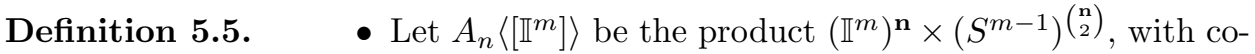
ordinates $\left(x_{i}\right) \times\left(u_{i j}\right)$.

- Let $C_{n}\left\langle\left[\mathbb{I}^{m}\right]\right\rangle$ be the closure of the image of $C_{n}\left(\mathbb{I}^{m}\right)$ under $\iota \times\left(\pi_{i j}\right)$, where $\iota$ is the inclusion of $C_{n}\left(\mathbb{I}^{m}\right)$ in $\left(\mathbb{I}^{m}\right)^{\mathbf{n}}$.

- Let $C_{n}\left\langle\left[\mathbb{I}^{m}, \partial\right]\right\rangle$ be the closure in $C_{n+2}\left\langle\left[\mathbb{I}^{m}\right]\right\rangle$ of the subspace of $C_{n+2}\left(\mathbb{I}^{m}\right)$ with $x_{1}=*_{+}=(0, \ldots, 0,1)$ and $x_{n+2}=*_{-}$.

In [37] we study $C_{n}\left\langle\left[\mathbb{I}^{m}\right]\right\rangle$ by relating it to the canonical compactification $C_{n}\left[\mathbb{I}^{m}\right]$, which is a manifold with corners. We characterize $C_{n}\left\langle\left[\mathbb{I}^{m}\right]\right\rangle$ as a subspace of its defining ambient space, as stated for $\widetilde{C}_{n}\left\langle\left[\mathbb{R}^{k}\right]\right\rangle$ in Theorem 4.4. The following is essentially Theorem 5.14 of [37.

Theorem 5.6. $C_{n}\left\langle\left[I^{k}\right]\right\rangle$ is the subspace of $\left(x_{i}\right) \times\left(u_{i j}\right)$ such that $\left(u_{i j}\right) \in \widetilde{C}_{n}\left\langle\left[\mathbb{R}^{k}\right]\right\rangle$ and if $x_{i} \neq x_{j}$, then $u_{i j}$ is $u\left(x_{j}-x_{i}\right)$.

In our models, we need diagonal maps between configuration spaces. The idea is to add a point "infinitesimally far" from one point in a configuration, but to do so entails choosing a unit tangent vector at that point.

Definition 5.7. Let $C_{n}^{\prime}\left\langle\left[\mathbb{I}^{m}\right]\right\rangle=C_{n}\left\langle\left[\mathbb{I}^{m}\right]\right\rangle \times\left(S^{m-1}\right)^{\mathbf{n}}$. Let $A_{n}^{\prime}\left\langle\left[\mathbb{I}^{m}\right]\right\rangle=\left(\mathbb{I}^{m} \times\right.$ $\left.S^{m-1}\right)^{\mathbf{n}} \times\left(S^{m-1}\right)^{\left(\begin{array}{c}\mathbf{n} \\ 2\end{array}\right)}$, which is canonically diffeomorphic to $\left(\mathbb{I}^{m}\right)^{\mathbf{n}} \times\left(S^{m-1}\right)^{\frac{\mathbf{n}(\mathbf{n}+1)}{2}}$. We use coordinates for this latter presentation of the form $\left(x_{i}\right) \times\left(u_{i j}\right)$ with $i \leq j$, and if $i>j$ we set $u_{i j}=-u_{j i}$.

As in Theorem 4.5 we define maps between the $C_{n}^{\prime}\left\langle\left[\mathbb{I}^{m}\right]\right\rangle$ at the level of the ambient spaces $A_{n}^{\prime}\left\langle\left[\mathbb{I}^{m}\right]\right\rangle$, using Theorem $[5.6$ to check that they restrict appropriately. We are aided by the following combinatorial shorthand.

Definition 5.8. - Given a map of sets $\sigma: R \rightarrow S$ let $p_{\sigma}^{X}$, or just $p_{\sigma}$, denote the map from $X^{S}$ to $X^{R}$ which sends $\left(x_{i}\right)_{i \in S}$ to $\left(x_{\sigma(j)}\right)_{j \in R}$.

- Given $\sigma: \mathbf{m} \rightarrow \mathbf{n}$, define $A_{\sigma}: A_{n}^{\prime}\left\langle\left[\mathbb{I}^{m}\right]\right\rangle \rightarrow A_{m}^{\prime}\left\langle\left[\mathbb{I}^{m}\right]\right\rangle$ as $p_{\sigma}^{\mathbb{I}^{m}} \times p_{\sigma^{(2)}}^{S^{k-1}}$, where $\sigma^{(2)}=\sigma \times\left.\sigma\right|_{\left(\begin{array}{c}\mathbf{n} \\ 2\end{array}\right)}$.

Proposition 5.9 (Proposition 6.6 of [37]). The restriction $A_{\sigma}$ to $C_{n}^{\prime}\left\langle\left[\mathbb{I}^{m}\right]\right\rangle$ maps to $C_{m}^{\prime}\left\langle\left[\mathbb{I}^{m}\right]\right\rangle$. If $\sigma$ sends $1 \rightarrow 1$ and $n \rightarrow m$, then $A_{\sigma}$ also restricts to a map, which we call $F_{\sigma}$, from $C_{n-2}^{\prime}\langle[\mathbb{I}, \partial]\rangle$ to $C_{m-2}^{\prime}\langle[\mathbb{I}, \partial]\rangle$.

We may now define diagonal maps on compactified configuration spaces with tangential data.

Definition 5.10. Let $\delta^{i}: C_{n}^{\prime}\left\langle\left[\mathbb{I}^{m}, \partial\right]\right\rangle \rightarrow C_{n+1}^{\prime}\left\langle\left[\mathbb{I}^{m}, \partial\right]\right\rangle$ be $F_{\sigma_{i}}$ where $\sigma_{i}: \underline{n+3} \rightarrow$ $\underline{n+2}$ sends $j$ to itself if $j \leq i$ or $j-1$ if $j>i$.

A final key property of this compactification is that it is functorial for embeddings. The proof of the following theorem is identical to that of Corollary 4.8 of [37, using Theorem 5.8 of [37] and the fact that $C_{n}\langle[\mathbb{I}]\rangle=\Delta^{n}$. Recall that for a non-zero vector $v \in \mathbb{R}^{m}, u(v)=\frac{v}{\|v\|}$.

Theorem 5.11. For an embedding $f: \mathbb{I} \rightarrow \mathbb{I}^{k}$ there is an evaluation map ev $n(f)$ : $\Delta^{n} \rightarrow C_{n}\left\langle\left[\mathbb{I}^{k}\right]\right\rangle$ which extends the map from the interior of $\Delta^{n}$ to $C_{n}^{\prime}\left(\mathbb{I}^{k}\right)$ sending $\left(t_{i}\right)$ to $\left(f\left(t_{i}\right)\right) \times\left(u\left(f^{\prime}\left(t_{i}\right)\right)\right)$. 
One of the main themes of [36] and of [43] is connecting this evaluation map with the calculus of embeddings. Applying this calculus to embeddings of the unit interval is simpler than to embeddings of higher-dimensional manifolds because the category $\mathcal{U}_{k}(\mathbb{I})$ may be replaced by the category of subsets of a finite set (see Definition 2.7).

Definition 5.12. Let $\mathcal{D}_{k}^{m}$ be the functor from $P_{0}(k)$ to spaces which sends $S \subseteq[k]$ to $C_{\# S-1}^{\prime}\left\langle\left[\mathbb{I}^{m}, \partial\right]\right\rangle$ and which sends the inclusion $S \subset S \cup j$ to the map $\delta^{i}$ where $i$ is the number of elements of $S$ less than $j$. Let $D_{k}^{m}=\operatorname{holim} \mathcal{D}_{k}^{m}$.

In the notation of [36, $\mathcal{D}_{k}^{m}$ would be $\mathcal{D}_{k}\left\langle\left[\mathbb{I}^{m}\right]\right\rangle$. Because the realization of $P_{0}(k)$ is $\Delta^{k}$ and all of the maps $\delta^{i}$ are inclusions of subspaces, $D_{k}^{m}$ is a subspace of $\operatorname{Maps}\left(\Delta^{k}, C_{k}^{\prime}\left\langle\left[\mathbb{I}^{m}, \partial\right]\right\rangle\right)$. If $f \in \operatorname{Emb}\left(\mathbb{I}, \mathbb{I}^{m}\right)$ is a knot, $e v_{k}(f)$ defines an element of $D_{k}^{m}$, as we may simply check that if $t_{j}=t_{j+1}$ for some point $\left(t_{i}\right) \in \Delta^{k}$, then the image of $e v_{k}(f)\left(\left(t_{i}\right)\right)$ is in the image of $\delta^{j}$. By abuse, let $e v_{k}$ denote the adjoint map from $\operatorname{Emb}\left(\mathbb{I}, \mathbb{I}^{m}\right)$ to $D_{k}^{m}$. Building on the simpler "cutting method" definition of $T_{k} \operatorname{Emb}\left(\mathbb{I}, \mathbb{I}^{m}\right)$, as described in Section 3 of [36, Lemma 5.18 and the proof of Theorem 5.3 of [36, establishes the following.

Theorem 5.13. $D_{k}^{m}$ is homotopy equivalent to $T_{k} \operatorname{Emb}\left(\mathbb{I}, \mathbb{I}^{m}\right)$, and ev $v_{k}$ agrees with $\eta_{k}$ in the homotopy category.

We next take a similar point of view for immersions of an interval, in order to arrive at our model for $E_{m}$. This point of view is taken in Section 5.3 of [43, which also considers the homotopy fiber of the map from knots to immersions. Indeed, our Theorem 5.15] and Proposition 5.16 below overlap significantly with Propositions 5.12 and 5.13 of 43 .

Definition 5.14. - Let $d^{i}:\left(S^{m-1}\right)^{j} \rightarrow\left(S^{m-1}\right)^{j+1}$ be the $i$ th diagonal inclusion, which on coordinates repeats the $i$ th entry. By convention, for $i=0$ and $i=j+1$ we insert the basepoint $*_{S}$ as the first, respectively last, coordinate.

- Let $\mathcal{G}_{k}^{m}$ be the functor from $P_{0}(k)$ to spaces which sends $S \subseteq[k]$ to $\left(S^{m-1}\right)^{\# S-1}$ and which sends the inclusion $S \subset S \cup j$ to the diagonal map $d^{i}$ where $i$ is the number of elements of $S$ less than $j$.

- Let $G_{k}^{m}=\operatorname{holim} \mathcal{G}_{k}^{m}$.

As was true for $D_{k}^{m}, G_{k}^{m}$ is a subspace of the space of maps from $\Delta^{k}$ to the terminal space of $\mathcal{G}_{k}^{m}$, namely $\left(S^{m-1}\right)^{k}$. The evaluation map for immersions is the unit derivative map. By abuse, let $e v_{k}: \operatorname{Imm}\left(\mathbb{I}, \mathbb{I}^{m}\right) \rightarrow G_{k}^{m}$ send $f$ to the map which sends $t_{1}, \ldots, t_{k}$ to $u f^{\prime}\left(t_{1}\right), \ldots, u f^{\prime}\left(t_{k}\right)$.

Theorem 5.15. If $k \geq 1, G_{k}^{m}$ is homotopy equivalent to $T_{k} \operatorname{Imm}\left(\mathbb{I}, \mathbb{I}^{m}\right)$, and thus to $\operatorname{Imm}\left(\mathbb{I}, \mathbb{I}^{m}\right)$. Moreover, ev $v_{k}$ agrees with $\eta_{k}$ in the homotopy category.

Sketch of proof. There are many ways to establish this theorem. By the HirschSmale theorem [39], $\operatorname{Imm}\left(\mathbb{I}, \mathbb{I}^{m}\right)$ is homotopy equivalent to $\Omega S^{m-1}$, through the unit derivative map. But $e v_{1}: \operatorname{Imm}\left(\mathbb{I}, \mathbb{I}^{m}\right) \rightarrow \operatorname{holim}\left(* \rightarrow S^{m-1} \leftarrow *\right)$ is also the unit derivative map, which establishes the theorem for $k=1$. For the other $k$, we may use Lemma 2.9. since $\mathcal{G}_{k}^{m}$ is the pull-back of the standard cosimplicial model for $\Omega S^{m-1}$ through the functor $c_{k}$ of Definition 2.8. The $k$ th totalization of this cosimplicial model, which is fibrant, is homeomorphic to $\Omega S^{m-1}$ if $k \geq 1$, 
from which it follows that $\mathcal{G}_{k}^{m}$ is homotopy equivalent to $\Omega S^{m-1}$. The map from $\Omega S^{m-1}$ to the $k$ th totalization, and thus $G_{k}^{m}$, is through evaluation of the unit derivative.

Let $\tau: \operatorname{Emb}\left(\mathbb{I}, \mathbb{I}^{m}\right) \rightarrow \operatorname{Imm}\left(\mathbb{I}, \mathbb{I}^{m}\right)$ denote the inclusion. Let $\rho_{k}^{m}: \mathcal{D}_{k}^{m} \rightarrow \mathcal{G}_{k}^{m}$ denote the map of diagrams defined on each entry by projection from $C_{n}^{\prime}\left\langle\left[\mathbb{I}^{m}, \partial\right]\right\rangle=$ $C_{n}\left\langle\left[\mathbb{I}^{m}, \partial\right]\right\rangle \times\left(S^{m-1}\right)^{n}$ onto $\left(S^{m-1}\right)^{n}$, and let $p_{k}^{m}$ also denote the induced map on homotopy limits.

Proposition 5.16. The square

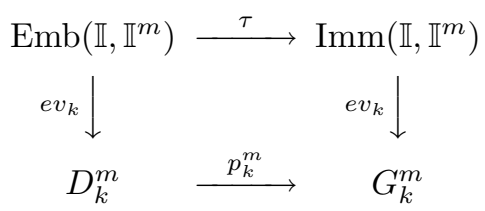

commutes. Moreover, $p_{k}^{m}$ agrees with $T_{k}(\tau)$ in the homotopy category.

Sketch of proof. The commutativity of the diagram is immediate from the definitions. That $p_{k}^{m}$ agrees with $T_{k}(\tau)$ in the homotopy category ultimately follows from the fact that for $U$ a disjoint union of $k+2$ open intervals, two of which contain endpoints of $\mathbb{I}$ and thus are fixed at one end, we have $\operatorname{Emb}\left(U, \mathbb{I}^{m}\right) \simeq C_{k}^{\prime}\left\langle\left[\mathbb{I}^{m}, \partial\right]\right\rangle$, $\operatorname{Imm}\left(U, \mathbb{I}^{m}\right) \simeq\left(S^{m-1}\right)^{k}$ and the inclusions from embeddings to immersions coincide with projection, as in the definition of $p_{k}^{m}$.

\subsection{A closer look at $E_{m}$.}

Proposition 5.17. The inclusion $\tau: \operatorname{Emb}\left(\mathbb{I}, \mathbb{I}^{m}\right) \rightarrow \operatorname{Imm}\left(\mathbb{I}, \mathbb{I}^{m}\right)$ is null-homotopic, so

$$
E_{m} \simeq \operatorname{Emb}\left(\mathbb{I}, \mathbb{I}^{m}\right) \times \Omega \operatorname{Imm}\left(\mathbb{I}, \mathbb{I}^{m}\right) \simeq \operatorname{Emb}\left(\mathbb{I}, \mathbb{I}^{m}\right) \times \Omega^{2} S^{m-1} .
$$

Proof. Given $f \in \operatorname{Emb}\left(\mathbb{I}, \mathbb{I}^{m}\right)$ consider the map $\rho(f): \Delta^{2} \rightarrow S^{m-1}$ which sends $t_{1}, t_{2}$ to either $u\left(f\left(t_{2}\right)-f\left(t_{1}\right)\right)$ if $t_{1} \neq t_{2}$ or $u\left(f^{\prime}(t)\right)$ if $t_{1}=t_{2}=t$. We may view $\rho(f)$ as a homotopy between $e v_{1}(f)$, which is the restriction to the $t_{1}=t_{2}$ edge, and the restriction to the $t_{1}=0$ and $t_{2}=1$ edges. But the restriction to these latter two edges is canonically null-homotopic, since their images lie in the southern hemisphere of $S^{m-1}$. Thus, $e v_{1}$ restricted to $\operatorname{Emb}\left(\mathbb{I}, \mathbb{I}^{m}\right)$ is null-homotopic. Since $e v_{1}$ is an equivalence on $\operatorname{Imm}\left(\mathbb{I}, \mathbb{I}^{m}\right)$ this implies that the inclusion of $\operatorname{Emb}\left(\mathbb{I}, \mathbb{I}^{m}\right)$ is null-homotopic.

That $E_{m} \simeq \operatorname{Emb}\left(\mathbb{I}, \mathbb{I}^{m}\right) \times \Omega \operatorname{Imm}\left(\mathbb{I}, \mathbb{I}^{m}\right)$ is immediate from its definition as the homotopy fiber of this inclusion, and that this is in turn weakly equivalent to $\operatorname{Emb}\left(\mathbb{I}, \mathbb{I}^{m}\right) \times \Omega^{2} S^{m-1}$ follows from the Hirsch-Smale theorem 39 .

The elements of $E_{3}$, which by the above is homotopy equivalent to $\operatorname{Emb}\left(\mathbb{I}, \mathbb{I}^{3}\right) \times$ $\Omega^{2} S^{2}$, are naturally equipped with framings.

Proposition 5.18 (Proposition 5.14 from [43]). The components of $E_{3}$ are canonically identified with isotopy classes of framed knots whose framing is even.

Given an element of $E_{3}$, a knot with a homotopy through immersions to the unknot, Volic "carries" the zero framing on the unknot through the homotopy to define a framing number on the knot, which is necessarily even. Note that $E_{m}$ is also the homotopy fiber of the inclusion of the space of framed embeddings in the space of framed immersions. When $m=3$ the space of framed immersions is 
homotopy equivalent to $\Omega S O(3)$, so the long-exact sequence in homotopy for this fibration reads

$$
\cdots \pi_{2}(S O(3))=0 \rightarrow \pi_{0}\left(E_{3}\right) \rightarrow \pi_{0} \operatorname{Emb}\left(\mathbb{I}, \mathbb{I}^{3}\right) \times \mathbb{Z} \rightarrow \pi_{1}(S O(3)) \cong \mathbb{Z} / 2 \rightarrow 0,
$$

consistent with the calculation that $\pi_{0}\left(F_{3}\right) \cong \pi_{0}\left(\operatorname{Emb}\left(\mathbb{I}, \mathbb{I}^{3}\right) \times 2 \mathbb{Z}\right.$.

\section{The MAin RESUlt}

We assemble our work to this point to prove the main result. As needed for the calculus of functors, extend $E_{m}$ to be a functor on the open sets of $\mathbb{I}$ by sending $U$ to the homotopy fiber of the inclusion $\operatorname{Emb}\left(U, \mathbb{I}^{m}\right) \rightarrow \operatorname{Imm}\left(U, \mathbb{I}^{m}\right)$. We will recover models for $E_{m}$ from those for embeddings and immersion spaces.

Lemma 6.1. If $A$ and $B$ are two functors from $\mathcal{U}(M)$ to spaces with a natural transformation $\tau$ between them, and $F$ is defined by $F(U)=$ hofib $(\tau: A(U) \rightarrow$ $B(U))$, then $T_{k}(F)=$ hofib $\left(T_{k}(A) \rightarrow T_{k}(B)\right)$.

Proof. The equality is immediate from the definition of $T_{k}$, since taking homotopy fibers commutes with taking homotopy limits.

In defining a fiber to $\rho_{k}^{m}$ we are led to the following.

Definition 6.2. Let $e_{i}: A_{n}\left\langle\left[\mathbb{I}^{m}\right]\right\rangle \rightarrow A_{n+1}\left\langle\left[\mathbb{I}^{m}\right]\right\rangle$ send $\left(u_{j \ell}\right)$ to $\left(v_{j \ell}\right)$ where $v_{i, i+1}=$ $*_{S}$, the basepoint of $S^{m-1}$ and the other $v_{j \ell}$ are equal to $u_{\sigma_{i}(j) \sigma_{i}(\ell)}$. As before $\sigma_{i}(j)=j$ or $j-1$ if $j<i$ or $j>i$ respectively. By abuse, use $e_{i}$ to denote its restriction to $C_{n}\left\langle\left[\mathbb{I}^{m}, \partial\right]\right\rangle$ mapping to $C_{n+1}\left\langle\left[\mathbb{I}^{m}, \partial\right]\right\rangle$, as one can check using Theorem [5.6.

Alternately, $e_{i}: C_{n}\left\langle\left[\mathbb{I}^{m}, \partial\right]\right\rangle \rightarrow C_{n+1}\left\langle\left[\mathbb{I}^{m}, \partial\right]\right\rangle$ is the restriction of $\delta^{i}$ to $C_{n}\left\langle\left[\mathbb{I}^{m}, \partial\right]\right\rangle$ $\times\left(*_{S}\right)^{n} \subset C_{n}^{\prime}\left\langle\left[\mathbb{I}^{m}, \partial\right]\right\rangle$.

Definition 6.3. Let $\mathcal{F}_{k}^{m}$ be the functor from $P_{0}(k)$ to spaces which sends $S \subseteq[k]$ to $C_{\# S-1}\left\langle\left[\mathbb{I}^{m}, \partial\right]\right\rangle$ and which sends the inclusion $S \subset S \cup j$ to the map $e^{i}$ where $i$ is the number of elements of $S$ less than $j$. Let $F_{k}^{m}=\underset{\operatorname{holim}}{\mathcal{F}_{k}^{m}}$.

Theorem 6.4. $F_{k}^{m}$ is homotopy equivalent to $T_{k} E_{m}$. For $m>3, \eta_{\infty}: E_{m} \rightarrow$ holim $T_{k} E_{m}$ is a weak equivalence.

Proof. We use the models $D_{k}^{m}$ and $G_{k}^{m}$ for $T_{k}$ Emb and $T_{k} \operatorname{Imm}$ as given in Theorems 5.13 and 5.15 respectively. By Proposition 5.16, $p_{k}^{m}: D_{k}^{m} \rightarrow G_{k}^{m}$ agrees with $T_{k}$ of the inclusion from embeddings to immersions. Applying Lemma 6.1 with $A=\operatorname{Emb}\left(-, \mathbb{I}^{m}\right), B=\operatorname{Imm}\left(-, \mathbb{I}^{m}\right)$, and the natural transformation between them being the standard inclusion, we have that $T_{k} E_{m}=$ hofib $p_{k}^{m}$.

If a map of diagrams indexed by $P_{0}(k)$ is a fibration object-wise, then the induced map on homotopy limits is a fibration and the fiber is given by the homotopy limit of the fibers object-wise (see, for example, Lemma 3.5 of [9]). Because $\rho_{k}^{m}$ is a fibration object-wise, we identify hofib $p_{k}^{m}=$ hofib (holim $\rho_{k}^{m}$ ) with such a homotopy limit of object-wise fibers. By our definition, the diagram of fibers is $\mathcal{F}_{k}^{m}$, whose homotopy limit is $F_{k}^{m}$, establishing the first half of the theorem.

The second half of the theorem is immediate from Theorems 5.3 and 5.4

Because of Proposition 5.17, we could alternately extend $E_{m}$ to a functor on $\mathcal{U}(\mathbb{I})$ by setting $E_{m}^{!}(U)=\operatorname{Emb}\left(U, \mathbb{I}^{m}\right) \times \Omega \operatorname{Imm}\left(U, \mathbb{I}^{m}\right)$. The extension $E_{m}^{!}$would lead to a set of approximations to $E_{m}$ different from the $F_{k}^{m}$. 
Recall from Proposition 4.7 that $\mathcal{K}_{m}$ is an operad with multiplication, which using Definition 2.17 has an associated cosimplicial object. We translate from $F_{k}^{m}$ to $\widehat{\operatorname{Tot}}\left(\mathcal{K}_{m}^{\bullet}\right)$, essentially through the standard projection $C_{n}\left\langle\left[\mathbb{I}^{m}, \partial\right]\right\rangle \rightarrow \widetilde{C_{n}}\left\langle\left[\mathbb{R}^{m}\right]\right\rangle$. We modify both $C_{n}\left\langle\left[\mathbb{I}^{m}, \partial\right]\right\rangle$ and this projection to define a natural transformation.

Definition 6.5. $\quad$ Let $\varepsilon \leq \frac{1}{6}$. For $x \in \mathbb{R}^{m}$, let $d_{+}(x)$ be the distance in $\mathbb{R}^{m}$ from $x$ to $*_{+}=(0, \ldots, 0,1)$ and $d_{-}(x)$ be the distance to $*_{-}$. Let $\gamma_{j}: \mathbb{R}^{m} \rightarrow \mathbb{R}$ be projection onto the $j$ th coordinate.

- Let $C_{n}\left\langle\left[\mathbb{I}^{m}, \partial_{\varepsilon}\right]\right\rangle$ be the subspace of $\left(x_{i}\right) \times\left(u_{i j}\right) \in C_{n}\left\langle\left[\mathbb{I}^{m}, \partial\right]\right\rangle$ where if $d_{+}\left(x_{i}\right)$ and $d_{+}\left(x_{j}\right)$ are less than $\varepsilon$ and $i<j$, then $\gamma_{k}\left(x_{i}\right)=\gamma_{k}\left(x_{j}\right)$ for $k<m$ and $\gamma_{m}\left(x_{i}\right) \geq \gamma_{m}\left(x_{j}\right)$. Moreover, if $x_{i}=x_{j}$ and $i<j$, then $u_{i j}=*_{S}$.

- Let $\mathcal{F}_{k, \varepsilon}^{m}$ be the functor from $P_{0}(k)$ to spaces which sends $S \subseteq[k]$ to $C_{\# S-1}\left\langle\left[\mathbb{I}^{m}, \partial_{\varepsilon}\right]\right\rangle$ and which sends the inclusion $S \subset S \cup j$ to the map $e^{i}$ where $i$ is the number of elements of $S$ less than $j$.

- Let $F_{k, \varepsilon}^{m}=\underset{\operatorname{holim}}{\longleftarrow} \mathcal{F}_{k, \varepsilon}^{m}$.

Proposition 6.6. The map $F_{k, \varepsilon}^{m} \rightarrow F_{k}^{m}$, induced by the natural transformation $\iota: \mathcal{F}_{k, \varepsilon}^{m} \rightarrow \mathcal{F}_{k}^{m}$ which at each entry is the canonical inclusion, is a homotopy equivalence.

Proof. It suffices to check that $\iota$ is a homotopy equivalence object-wise, for which we adapt the machinery developed in [37] for compactified configuration spaces. Both $C_{k}\left\langle\left[\mathbb{I}^{m}, \partial_{\varepsilon}\right]\right\rangle$ and $C_{k}\left\langle\left[\mathbb{I}^{m}, \partial\right]\right\rangle$ are quotients of the canonical Axelrod-Singer compactifications, which we call $C_{k}\left[\mathbb{I}^{m}, \partial_{\varepsilon}\right]$ and $C_{k}\left[\mathbb{I}^{m}, \partial\right]$ respectively; see Definitions 1.3 and 4.18 of [37] for the definition of $C_{k}\left[\mathbb{I}^{m}, \partial\right]$, which can be modified as in Definition [6.5 for $C_{k}\left[\mathbb{I}^{m}, \partial_{\varepsilon}\right]$. These quotient maps are homotopy equivalences, by the proof of Theorem 5.10 of [37, which applies verbatim in these cases.

$C_{k}\left[\mathbb{I}^{m}, \partial_{\varepsilon}\right]$ retracts to its subspace $C_{k}\left[\mathbb{I}^{m}-N_{ \pm}^{\varepsilon}\right]$, where $N_{ \pm}^{\varepsilon}$ is the union of the $\varepsilon$ neighborhoods of $*_{+}$and $*_{-}$by scaling the $x_{i}$ by $1-\varepsilon$. Both $C_{k}\left[\mathbb{I}^{m}, \partial\right]$ and $C_{k}\left[\mathbb{I}^{m}-N_{ \pm}^{\varepsilon}\right]$ are manifolds with corners (see Theorem 4.4 of [37]), and thus are homotopy equivalent to their interiors, $C_{k}\left(\operatorname{Int}\left(\mathbb{I}^{m}\right)\right)$ and $C_{k}\left(\operatorname{Int}\left(\mathbb{I}^{m}-N_{ \pm}^{\varepsilon}\right)\right)$ respectively. But these interior configuration spaces are diffeomorphic, since $\operatorname{Int}\left(\mathbb{I}^{m}\right)$ and $\operatorname{Int}\left(\mathbb{I}^{m}-N_{ \pm}^{\varepsilon}\right)$ are. Composing this diffeomorphism with the previous homotopy equivalences establishes the equivalence of $C_{k}\left\langle\left[\mathbb{I}^{m}, \partial_{\varepsilon}\right]\right\rangle$ and $C_{k}\left\langle\left[\mathbb{I}^{m}, \partial\right]\right\rangle$ and thus establishes the result.

We use $C_{k}\left\langle\left[\mathbb{I}^{m}, \partial_{\varepsilon}\right]\right\rangle$ because they readily project to $\widetilde{C_{k}}\left\langle\left[\mathbb{R}^{m}\right]\right\rangle$ in a way compatible with its cosimplicial structure maps.

Definition 6.7. - Let $\left(a_{i}\right)_{i=1}^{m}, a_{i} \in \mathbb{R}$ denote a point in $\mathbb{R}^{m}$. Define $\lambda_{+}$: $\left(\mathbb{R}^{m}-*_{+}\right) \rightarrow \mathbb{R}^{m}$ by sending $\left(a_{i}\right)$ to $\left(b_{i}\right)$ where if $i \neq m$, then $b_{i}=a_{i}$ and

$$
b_{m}= \begin{cases}\frac{\varepsilon a_{m}}{d_{+}\left(a_{i}\right)} & d_{+}\left(a_{i}\right)<\varepsilon \\ a_{m} & d_{+}\left(a_{i}\right) \geq \varepsilon .\end{cases}
$$

Define $\lambda_{-}:\left(\mathbb{R}^{m}-*_{-}\right) \rightarrow \mathbb{R}^{m}$ similarly, and let $\lambda=\lambda_{+} \circ \lambda_{-}$.

- Define $\pi_{k}: C_{k}\left\langle\left[\mathbb{I}^{m}, \partial_{\varepsilon}\right]\right\rangle \rightarrow \widetilde{C_{k}}\left\langle\left[\mathbb{R}^{m}\right]\right\rangle \subset\left(S^{m-1}\right)^{\left(\begin{array}{l}k \\ 2\end{array}\right)}$ by sending $\left(x_{i}\right) \times\left(u_{i j}\right)$ to $\left(v_{i j}\right)$ where $v_{i j}$ is:

$$
-u\left(\lambda\left(x_{i}\right)-\lambda\left(x_{j}\right)\right) \text { if } x_{i} \neq x_{j} \text { and neither equals } *_{+} \text {or } *_{-} \text {; }
$$


- the Jacobian on $\lambda$ applied to $u_{i j}$ if $x_{i}=x_{j}$;

- $*_{S}$, if $x_{i} \neq x_{j}$ and either $x_{i}=*_{+}$or $x_{j}=*_{-}$.

Proposition 6.8. $\pi_{k}$ is continuous.

Proof. We first identify $\pi_{k}$ on the subspace $C_{k}\left\langle\left[\mathbb{I}^{m}-\left(*_{+} \cup *_{-}\right)\right]\right\rangle$with the composite of $C_{k}\langle[\lambda]\rangle$, the map on configuration spaces induced by the embedding $\lambda$ (see Corollary 4.8 of [37]), and the canonical projection $C_{k}\left\langle\left[\mathbb{R}^{m}\right]\right\rangle$ to $\widetilde{C_{k}}\left\langle\left[\mathbb{R}^{m}\right]\right\rangle$. What remains is to check continuity on the subspace in which some $x_{n}=*_{+}$. Consider a sequence $\left\{\left(x_{i}^{\ell}\right),\left(u_{i j}^{\ell}\right)\right\}_{\ell=1}^{\infty}$ with limit point $\left(x_{i}^{\infty}\right) \times\left(u_{i j}^{\infty}\right)$, so that $x_{n}^{\infty}=*_{+}$. We show that its image under $\pi_{k}$ has $v_{n j}$, which approaches $*_{S}$ if $x_{j}^{\infty} \neq *_{+}$or $n<j$ or which approaches $-*_{S}$ otherwise. For each $j$, either $x_{j}^{\infty} \in N_{+}^{\varepsilon}$, which is also true for $\ell$ sufficiently large, in which case $u_{n j}$ must be $*_{S}$ if $n<j$ or $-_{S}$ if $n>j$, so that the sequence $v_{n j}$ would be eventually constant at $*_{S}$ or $-*_{S}$. Or if $x_{j}^{\infty} \notin N^{\varepsilon}$, then as $x_{n}^{\ell} \mapsto *_{+}$the last coordinate of $\lambda_{+}\left(x_{i}^{\ell}\right)$ becomes arbitrarily large. Because $x_{j}^{\ell} \mapsto x_{j}$ stays in $\mathbb{I}^{m}$ we have $u\left(\lambda\left(x_{n}^{\ell}\right)-\lambda\left(x_{j}^{\ell}\right)\right) \mapsto *_{S}$. Continuity when some $x_{n}=*_{-}$works similarly.

We may now assemble our main result, Theorem 1.1 which casts the embedding calculus tower for $E_{m}$ in the language of operads. For convenience, we restate the theorem here.

Theorem 6.9. The $k$ th approximation to $E_{m}$ in the embedding calculus, namely $T_{k} E_{m}$, is weakly equivalent to $\widetilde{\operatorname{Tot}}^{k} \mathcal{K}_{m}^{\bullet}$.

Proof. We will check that the maps $\pi_{k}$ assemble to a natural transformation of functors from $\mathcal{F}_{k}^{m}$ to $\mathcal{K}_{m}^{\bullet} \circ c_{k}$, with $c_{k}$ as in Definition 2.8, which gives rise to a weak equivalence on homotopy limits. Theorem 6.4 then says that the homotopy limit of $\mathcal{F}_{k}^{m}$ is weakly equivalent to $T_{k} E_{m}$, and Lemma 2.9 implies that the homotopy limit of $\mathcal{K}_{m}^{\bullet} \circ c_{k}$ is weakly equivalent to $\widetilde{\operatorname{Tot}}^{k} \mathcal{K}_{m}^{\bullet}$, establishing the theorem.

For the assembled $\pi_{k}$ to be a natural transformation, we must have $\pi_{k} \circ e^{i}=$ $d^{i} \circ \pi_{k}$. For most $i$ this is immediate to check, as repeating coordinates and passing to the quotient $\widetilde{C}_{k}\left\langle\left[\mathbb{R}^{m}\right]\right\rangle$ are processes which clearly commute. The $i=0$ and $i=k+1$ cases require the modifications we made in Definition 6.7 For $\mathcal{K}_{m}^{\bullet} \circ c_{k}$ we trace through Definitions 2.17 and 3.1. Theorem 4.5 and Proposition 4.7 to see that $d^{k+1}$ takes a point $\left(u_{i j}\right) \in \widetilde{C}_{k}\left\langle\left[\mathbb{R}^{m}\right]\right\rangle$, leaves all these $u_{i j}$ unchanged, and adds $u_{i, k+1}=*_{S}$ for all $i$ to obtain a point in $\widetilde{C}_{k+1}\left\langle\left[\mathbb{R}^{m}\right]\right\rangle$. On the other hand, $e^{k+1}$ adds the $(k+1)$ st point to the configuration at $*_{-}$, which under $\pi_{k}$ will also lead to all $u_{i, k+1}=*_{S}$. The $i=0$ case works similarly.

The fact that the assembled $\pi_{k}$ induces a weak equivalence on homotopy limits follows from its being a homotopy equivalence object-wise. We already know from the proof of Proposition 6.6 that $C_{k}\left\langle\left[\mathbb{I}^{m}, \partial_{\varepsilon}\right]\right\rangle$ is homotopy equivalent to the subspace $C_{k}\left(\operatorname{Int}\left(\mathbb{I}^{m}-N_{ \pm}^{\varepsilon}\right)\right)$, which is diffeomorphic to $C_{k}\left(\mathbb{R}^{m}\right)$. Composed with this diffeomorphism on this subspace, $\pi_{k}$ is the standard projection $C_{k}\left(\mathbb{R}^{m}\right) \rightarrow \widetilde{C_{k}}\left(\mathbb{R}^{m}\right)$ followed by the canonical map to $\widetilde{C_{k}}\left\langle\left[\mathbb{R}^{m}\right]\right\rangle$, which is a homotopy equivalence by Corollaries 4.5 and 5.9 of 37 .

The first half of Corollary 1.2, which states that for $m>3$ the totalization of the Kontsevich operad faithfully models the weak homotopy type of $E_{m}$, now follows from Theorem 6.4. The second half of Corollary [1.2, which states that for $m=3$ 
all real-valued finite-type framed knot invariants are pulled back from this operad model, follows from the main results of 43. We may take any framed knot, double its framing, and then apply Proposition 5.18 to get a corresponding component of $E_{3}$. In [43, Volic uses Bott-Taubes integrals to define finite-type invariants on the Taylor tower for $E_{3}$, which we have now identified with the Tot tower for $\mathcal{K}_{3}$. See in particular Theorems 1.2 and 1.3 and Section 6.4 of [43].

\section{OBSERVATIONS AND CONSEQUENCES}

7.1. Spectral sequences. The results in this section parallel those of section 7 of [36]. Applying the homotopy spectral sequence of Proposition 2.10 for $\mathcal{K}_{m}^{\bullet}$ we immediately have the following.

Theorem 7.1. There is a spectral sequence converging to $\pi_{*}\left(\widetilde{\text { Tot }} \mathcal{K}_{m}^{\bullet}\right)$ with

$$
E_{1}^{-p, q}=\bigcap \operatorname{ker} s^{k} \subseteq \pi_{q}\left(C_{p}\left(\mathbb{R}^{m}\right)\right) .
$$

The $d_{1}$ differential is the restriction to this kernel of the map

$$
\sum_{i=0}^{p+1}(-1)^{i} d_{*}^{i}: \pi_{q}\left(C_{p-1}\left(\mathbb{R}^{m}\right)\right) \rightarrow \pi_{q}\left(C_{p}\left(\mathbb{R}^{m}\right)\right) .
$$

Theorem 6.4 implies that this spectral sequence computes homotopy groups of $E_{m}$ when $m \geq 4$. Except for in the $p=1$ column, this spectral sequence coincides exactly with that studied with rational coefficients in [34, so we do not give a more explicit description here. Kontsevich 23] has also examined the rows of this spectral sequence.

For $m=3$, the case of classical knots, we conjecture that $\eta_{k}: E_{m} \rightarrow T_{k} E_{m}$ is a universal type- $(k-1)$ framed knot invariant over the integers. For $k \leq 3$, we may deduce this from the main results of [9]. In unpublished work, Conant has shown that the entries $E_{-k, k}^{2}$ of this spectral sequence are isomorphic to the module of primitive weight systems of degree $k-1$ over the integers, a first step to this conjecture in full generality.

In light of Theorem 4.11, the homology spectral sequence from Theorem 2.11 has a pleasant description. Recall Definition 2.17, which for operads of vector spaces introduces the notation of $H H^{*}(\mathcal{O})$ for the total cohomology of the associated cosimplicial object.

Theorem 7.2. There is a spectral sequence with $E_{-p, q}^{2}=H H^{p, q}\left(\right.$ Poiss $\left._{m}\right)$ which for $m \geq 4$ converges to the homology of $\widetilde{\operatorname{Tot}} \mathcal{K}_{m}^{\bullet}$, and thus of $E_{m}$.

Proof. If we use the second description of the homology spectral sequence from Theorem 2.11, then $E_{-*, *}^{1}$ will be $H_{*}\left(\mathcal{K}_{m}^{\bullet}\right)$, which is the Poisson operad by Theorem 4.11. The induced operad with multiplication structure on the Poisson operad is the standard one. Thus, the $d^{1}$ differential will coincide with the differential for total cohomology of the Poisson operad, and the $E^{2}$-term will be the total (or Hochschild) cohomology of the Poisson operad as stated.

It remains to check the convergence conditions of Theorem 2.11. In the case of the Kontsevich operad, the entries $\mathcal{K}_{m}^{k}=\widetilde{C}_{k}\left\langle\left[\mathbb{R}^{m}\right]\right\rangle$ are homotopy equivalent to $C_{k}\left(\mathbb{R}^{m}\right)$, which are simply connected if $m \geq 3$. Using the first definition of Theorem 2.11, we start with $H_{*}\left(C_{p}\left(\mathbb{R}^{m}\right)\right)$ and explicitly understand the kernels of the maps $s_{*}^{i}$. We use Theorem 4.11 and Definition 4.10 to identify $H_{*}\left(C_{p}\left(\mathbb{R}^{m}\right)\right)$ in 
terms of products of brackets in variables $x_{1}, \ldots, x_{p}$. Tracing through the definitions of the associated cosimplicial object, $s^{i}$ sends a product of brackets in the $x_{j}$ to either zero, if the variable $x_{i}$ appears in a bracket, or the monomial obtained by removing $x_{i}$ and relabeling $x_{j}$ to $x_{j-1}$ for $j>1$, if $x_{i}$ does not appear in a bracket. Therefore to be in the kernel of all of the $s^{i}$, all of the variables $x_{i}$ must appear in a bracket, so there must be at least $\frac{k}{2}$ brackets, leading to a total degree of at least $\frac{k(m-1)}{2}$. For $m>3$, this is greater than $k$ and thus gives the estimate needed for application of Theorem 2.11.

This spectral sequence in real-valued cohomology coincides with the homotopy spectral sequence of the Taylor tower for the functor to spectra $U \mapsto \mathbb{R} \wedge E_{m}(U)$, the real Eilenberg-Mac Lane spectrum smashed with $E_{m}(U)$. For $m=3$, Volic's results 43, 44] imply that the map from the knot space to this Taylor tower serves as a universal framed finite-type invariant over the real numbers.

7.2. A little two-cubes action from the McClure-Smith framework. Theorem 1.1 fits perfectly into the framework created by McClure and Smith in their solution of the Deligne conjecture over the integers [28. One of their central results is the following.

Theorem 7.3. The totalization of the associated cosimplicial object of an operad with multiplication admits an action of an operad equivalent to the little 2-cubes operad, as does its homotopy invariant totalization.

Proof. We are simply collecting results from [28] and 29]. For the standard totalization, we are simply quoting Theorem 3.3 in [28]. For the homotopy invariant totalization, Theorem 15.3 of 29] says that Tot of any cosimplicial space with what is called a $\Xi^{2}$-structure has an action of an operad equivalent to the little 2-cubes. Proposition 10.3 of 29 identifies an operad with multiplication structure on a sequence of spaces with a $\Xi^{2}$ structure.

Example 7.4. Consider the cosimplicial model for the space of maps from $S^{2}$ to $X$, namely $X^{S_{\bullet}^{2}}$. By Theorem 3.3, $S_{\bullet}^{2} \cong \mathcal{B}^{\bullet}$, so there is an operad structure on this collection of spaces. In order to get an operad with multiplication, we restrict each $X^{\mathcal{B}^{n}}=\left(x_{\alpha}\right)$ to the subspace in which $x_{+}=*$, where + is the basepoint of $\mathcal{B}^{n}$ and * is the base point of $X$. The operad structure maps restrict appropriately, and we obtain $X_{\star}^{S_{\bullet}^{2}}$, to which the associative operad maps at each level to the point with all $x_{\alpha}=*$.

Applying Theorem 7.3, the totalization of $X_{\star}^{S_{\bullet}^{2}}$ is a little 2-cubes space, and we know that its totalization is $\Omega^{2} X$. McClure and Smith fully develop this example (more generally for the standard model of $\Omega^{n} X$ ) in Section 11 of [29]. They show that the little 2- (or $n$-) cubes action which arises in these examples coincides with the standard ones.

We can immediately establish Theorem 1.4, one of our main results, which we restate here.

Theorem 7.5. For any $m$, there is a little two-cubes action on $\widetilde{\operatorname{Tot}}\left(\mathcal{K}_{m}^{\bullet}\right)$. For $m>3, E_{m}$ is a two-fold loop space.

Proof. Applying Theorem 7.3 for the Kontsevich operad with its given multiplication establishes the two-cubes action. 
By Theorem 1.1, if $m \geq 4, E_{m}$ is homotopy equivalent to $\widetilde{\operatorname{Tot}}\left(\mathcal{K}_{m}^{\bullet}\right)$, so it has a 2-cubes action as well. But $E_{m}$ is connected for $m \geq 4$, since it is the product of $\Omega\left(\operatorname{Imm}\left(\mathbb{I}, \mathbb{I}^{m}\right)\right) \simeq \Omega^{2} S^{m-1}$ and $\operatorname{Emb}\left(\mathbb{I}, \mathbb{I}^{m}\right)$, which are both connected (that the latter space is connected is because any path through maps from an embedding to the standard one becomes an isotopy once put in general position). By the recognition theorem of [3], 4], $E_{m}$ is a 2-fold loop space.

Our operad model for $E_{m}$ has already been important for closer examination of the homotopy type of $E_{m}$. In 24], Volic and Lambrechts establish a formality result for this model, which determines the rational homotopy type of $E_{m}$ for $m>3$.

A two-cubes action has been used with spectacular success in dimension three. In [7, Budney constructs a little two-cubes action directly on the space of framed (long) knots in any dimension. He goes on to show that the two-cubes action is free when $m=3$, generated by the components of prime knots. In [8, he determines the homotopy types of these prime components in terms of the JSJ decompositions of prime knots.

In further work, we may develop a model for framed knots closely related to those studied in this paper. Namely, there is an operad whose entries are $\widetilde{C}_{n}\left\langle\left[\mathbb{R}^{m}\right]\right\rangle \times$ $(S O(m))^{n}$, where in the operad composition, the elements of $S O(m)$ act on the configurations (compare with the operad of "turning balls"). The techniques in this paper should also show that the totalization of this operad models spaces of framed knots. It may then be fruitful to compare Budney's two-cubes action to the one which arises from the McClure-Smith machinery, as well as to combinatorial product and bracket structures on homology defined by Tourtchine [40].

\section{ACKNOWLEDGEMENTS}

We thank J. McClure and J. Smith for their interest in this project, answers to questions, and especially for writing Section 15 of 29. We thank M. Markl and J. Stasheff for comments on early versions of this work, and M. Kontsevich for helpful conversations.

\section{REFERENCES}

[1] S. Axelrod and I. Singer. Chern-Simons perturbation theory, II. Jour. Diff. Geom. 39 (1994), no. 1, 173-213. MR.1258919 (95b:58163)

[2] A. J. Berrick, F. Cohen, Y. Wong and J. Wu. Configurations, braids, and homotopy groups, J. Amer. Math. Soc. (to appear).

[3] J. M. Boardman. Homotopy structures and the language of trees. In Algebraic Topology, Proc. Sympos. Pure Math, Vol. XXII, AMS, 1971, pp. 37-58. MR0350723 (50:3215)

[4] J. M. Boardman and R. Vogt. Homotopy invariant algebraic structures on topological spaces. Lecture Notes in Mathematics, Vol. 347, 1973. MR0420609 (54:8623a)

[5] A. Bousfield and D. Kan. Homotopy limits, completions and localizations. Lecture Notes in Mathematics, Vol. 304, 1972. MR0365573 (51:1825)

[6] A. Bousfield. On the homology spectral sequence of a cosimplicial space. Amer. J. Math. 109 (1987), no. 2, 361-394. MR0882428 (88j:55017)

[7] R. Budney. Little cubes and long knots. To appear in Topology.

[8] Topology of spaces of knots in dimension 3. math.GT/0506524.

[9] R. Budney, J. Conant, K. Scannell and D. Sinha. New perspectives on self-linking. Advances in Mathematics 191 (2005) 78-113. MR2102844 (2005h:57013)

[10] F. Cohen. The homology of $C_{n+1}$ spaces. In Lecture Notes in Mathematics 533 (1976).

[11] W. Fulton and R. MacPherson. Compactification of configuration spaces. Annals of Mathematics 139 (1994), 183-225. MR 1259368(95j:14002) 
[12] G. Gaiffi. Models for real subspace arrangements and stratified manifolds. Int. Math. Res. Not. (2003) no. 12, 627-656. MR.1951400(2004d:52021)

[13] M. Gerstenhaber and A. Voronov, Homotopy $G$-algebras and moduli space operads. Intern. Math. Res. Notices 3 (1995) 141-153. MR1321701 (96c:18004)

[14] E. Getzler and J. Jones. Operads, homotopy algebra and iterated integrals for double loop spaces. hep-th/9403055.

[15] T. Goodwillie. Calculus II, Analytic functors. K-Theory 5 (1991/92), no. 4, 295-332. MR 1162445 (93i:55015)

[16] T. Goodwillie and M. Weiss. Embeddings from the point of view of immersion theory II. Geometry and Topology 3 (1999), 103-118. MR1694808(2000c:57055b)

[17] T. Goodwillie, J. Klein and M. Weiss. Spaces of smooth embeddings, disjunction and surgery. Surveys on surgery theory, Vol. 2, 221-284, Ann. of Math. Stud., 149 (2001). MR.1818775 (2001m:57053)

[18] T. Goodwillie, J. Klein and M. Weiss. A Haefliger type description of the embedding calculus tower. Topology 42 (2003), no. 3, 509-524. MR1953238(2003m:57062)

[19] T. Goodwillie and J. Klein. Excision statements for spaces of smooth embeddings. In preparation.

[20] T. Goodwillie. Excision estimates for spaces of homotopy equivalences. Preprint is available at: http://www.math.brown.edu/faculty/goodwillie.html.

[21] P. S. Hirschhorn, Model categories and their localizations, Math. Surveys Monographs, vol. 99, Amer. Math. Soc., Providence, 2003. MR1944041 (2003j:18018)

[22] M. Kontsevich, Operads and motives in deformation quantization, Lett. Math. Phys. 48 (1999) 35-72. MR 1718044 (2000j:53119)

[23] _ Operads of little discs in algebra and topology, Lecture at the Mathematical Challenges Conference, UCLA, 2000.

[24] P. Lambrechts and I. Volic. The rational homotopy type of spaces of knots in high codimension. In preparation.

[25] M. Markl. A compactification of the real configuration space as an operadic completion. $J$. Algebra 215 (1999), no. 1, 185-204. MR1684178 (2000g:55013)

[26] M. Markl, S. Shnider, and J. Stasheff. Operads in algebra, topology and physics. Math. Surv. and Monographs, 96. AMS, Providence, RI, 2002. MR1898414 (2003f:18011)

[27] J. P. May. The geometry of iterated loop spaces. Lecture Notes in Mathematics, Vol. 271, 1972. MR0420610 (54:8623b)

[28] J. McClure and J. Smith. A solution of Deligne's Hochschild cohomology conjecture. In: Recent progress in homotopy theory (Baltimore, MD, 2000), 153-193, Contemp. Math., 293, AMS, 2002. MR,1890736 (2003f:55013)

[29] Cosimplicial objects and little $n$-cubes. I. math.QA/0211368. Amer. J. Math. 126 (2004), 1109-1153. MR2089084 (2005g:55011)

[30] Operads and cosimplicial objects: an introduction. In Axiomatic, Enriched and Motivic Homotopy Theory. Edited by J.P.C. Greenlees. Kluwer 2004. MR2061854(2005b:55018)

[31] G. Mislin. Wall's finiteness obstruction. In Handbook of algebraic topology, edited by I. M. James. Elsevier, 1995. MR1361911 (97g:57029)

[32] R. Palais. Homotopy theory of infinite-dimensional manifolds. Topology 5 (1966) 1-16. MR0189028 (32:6455)

[33] D. Rector. Steenrod operations in the Eilenberg-Moore spectral sequence. Comment. Math. Helv. 45 (1970) 540-552. MR0278310(43:4040)

[34] K. Scannell and D. Sinha. A one-dimensional embedding complex. Journal of Pure and Applied Algebra 170 (2002) No. 1, 93-107. MR.1896343(2003b:55012)

[35] B. Shipley. Convergence of the homology spectral sequence of a cosimplicial space. Amer. J. Math. 118 (1996), no. 1, 179-207. MR:1375305 (97b:55023)

[36] D. Sinha. The topology of spaces of knots. math.AT/0202287.

[37] Manifold-theoretic compactifications of configuration spaces. Selecta Math. 10 (2004), 391-428. MR2099074 (2005h:55015)

[38] Configuration spaces, Hopf invariants, and Whitehead products. In preparation.

[39] S. Smale. The classification of immersions of spheres in Euclidean spaces. Annals of Mathematics (2) 69 (1959) 327-344. MR0105117 (21:3862)

[40] V. Tourtchine. On the homology of long knots. Advances in topological quantum field theory, NATO Conference Proceedings, Kluwer Acad. Publ., Dordrecht, 2004, pp. 23-52. MR2147415 
[41] V. Vassiliev. Complements of discriminants of smooth maps: topology and applications. Translations of Mathematical Monographs, 98. American Mathematical Society, Providence, RI, 1992. MR1168473 (94i:57020)

[42] - Topology of two-connected graphs and homology of spaces of knots. In: Differential and symplectic topology of knots and curves, 253-286, AMS Transl. Ser. 2, 190, 1999. MR 1738399 (2001b:55030)

[43] I. Volic. Finite type knot invariants and calculus of functors. To appear in Compositio Mathematica. math.AT/0401440.

[44] Configuration space integrals and Taylor towers for spaces of knots. math.GT/ 0401282.

[45] M. Weiss. Calculus of embeddings. Bulletin of the AMS 33 (1996), 177-187. MR.1362629 (96j:57035)

[46] Embeddings from the point of view of immersion theory I. Geometry and Topology 3 (1999), 67-101. MR1694812 (2000c:57055a)

Department of Mathematics, University of Oregon, Eugene, Oregon 97403 